\title{
Numerical Study of Traffic Pollutant Dispersion within Different Street Canyon Configurations
}

\author{
Yucong Miao, Shuhua Liu, Yijia Zheng, Shu Wang, and Yuan Li \\ Department of Atmospheric and Oceanic Sciences, School of Physics, Peking University, Beijing 100871, China \\ Correspondence should be addressed to Shuhua Liu; lshuhua@pku.edu.cn
}

Received 25 October 2013; Revised 30 December 2013; Accepted 28 January 2014; Published 13 March 2014

Academic Editor: Gwo-Fong Lin

Copyright (c) 2014 Yucong Miao et al. This is an open access article distributed under the Creative Commons Attribution License, which permits unrestricted use, distribution, and reproduction in any medium, provided the original work is properly cited.

\begin{abstract}
The objective of this study is to numerically study flow and traffic exhaust dispersion in urban street canyons with different configurations to find out the urban-planning strategies to ease the air pollution. The Computational Fluid Dynamics (CFD) model used in this study-Open Source Field Operation and Manipulation (OpenFOAM) software package-was firstly validated against the wind-tunnel experiment data by using three different $k-\varepsilon$ turbulence models. And then the patterns of flow and dispersion within three different kinds of street canyon configuration under the perpendicular approaching flow were numerically studied. The result showed that the width and height of building can dramatically affect the pollution level inside the street canyon. As the width or height of building increases, the pollution at the pedestrian level increases. And the asymmetric configuration (step-up or stepdown street canyon) could provide better ventilation. It is recommended to design a street canyon with nonuniform configurations. And the OpenFOAM software package can be used as a reliable tool to study flows and dispersions around buildings.
\end{abstract}

\section{Introduction}

Street canyon is a typical configuration of densely built urban areas, referring to a narrow street between buildings that line up continuously along both sides. It has a different climate where microscale meteorological processes dominate [1]. Air exchange between street level and the atmospheric wind above the roof level is limited inside the street canyon, and the near ground vehicular pollutant emissions are not effectively diluted and removed, which considerably deteriorates the pedestrian level air quality in the street canyon and imposes harmful impacts on the health of the city population. Thus, it is necessary to study how the pollutants are transported and distributed in the street canyons and find out some urbanplanning strategies to ease the air pollution inside the street canyon.

The primary methods to study the flow fields and pollutant dispersion within the street canyon include field measurements, laboratory physical experiments, and numerical simulation. Field measurements can offer lots of useful information on the flow fields and pollutant concentration distribution within the actual street canyons [2-4]. However, field measurements are limited by their low spatial resolution, high cost, and uncontrollable meteorological conditions [5].

In addition to the field measurements and observations, the laboratory physical experiments (water tank or windtunnel experiments) have also been extensively applied to understand the complex flow and pollutant distribution patterns within street canyons under fully controllable upwind boundary conditions for airflow [6-11]. However, the physical experiments provide data only for a few number of discrete measurement points.

Comparing with these two experimental methods mentioned above, the numerical simulation, a relatively economic approach, allows us to visualize the airflow and pollutant concentration fields where measurements are not available. It can provide more comprehensive information on the flow fields and pollutants dispersion within the street canyon, with high spatial and temporal resolutions. And the Computational Fluid Dynamics (CFD) models are now widely used to 


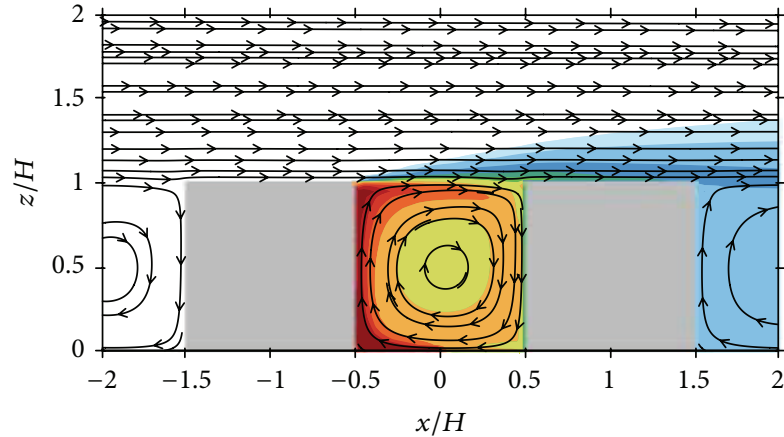

(a)

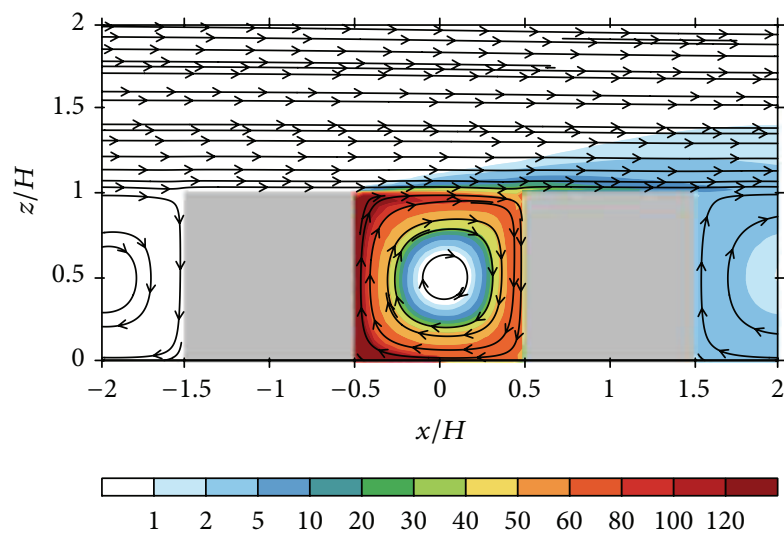

(c)

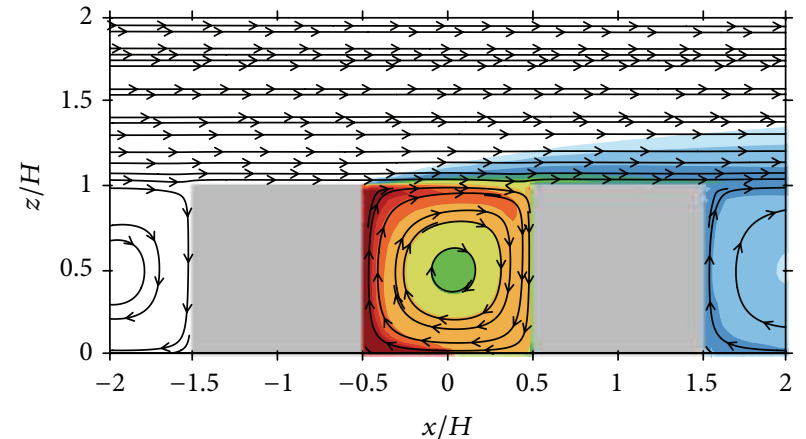

(b)

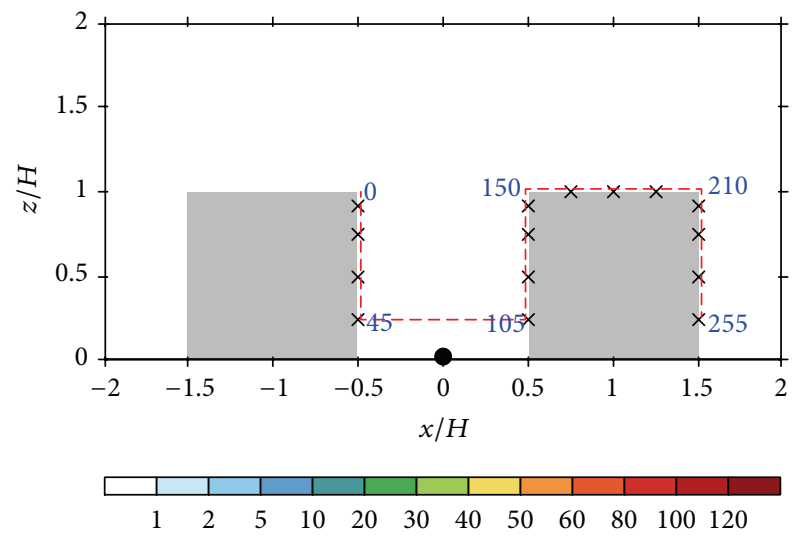

(d)

FIGURE 1: The simulated normalized concentration distributions and the velocity streamline patterns of different $k$ - $\varepsilon$ turbulence models. (a) The standard $k-\varepsilon$ model, (b) the RNG $k-\varepsilon$ model, and (c) the realizable $k-\varepsilon$ model. The locations of measurement are indicated by the crosses in (d), and the source is shown by the black dot in the middle of the street canyon. The red dash line plot in (d) indicates the route of sampling for validation, while the blue number shows the distance $(\mathrm{mm})$ along the sampling route from the upper corner point $(x=-0.5 H$ and $z=1 H)$ of the leeward side of the upstream building.

simulate airflow and pollutant dispersion in the street canyon [12-23]. However, most of the previous CFD simulations of the street canyon are two-dimensional [12-18]: hence the accuracy of these simulations is limited. And most of the previous works focused on the symmetric street canyon and the effect of the street aspect ratio (i.e., ratio of the building height to the street canyon width), while the asymmetric street canyon and other factors of street canyon geometry were not fully recognized. The objective of this work is to find out some crucial parameters of street canyon, other than the street aspect ratio, which could be used to ease the pollution in the street canyon.

In this study, a three-dimensional CFD model was used to better understand the pollutant transport process and distribution patterns in the street canyons with three different kinds of configuration. The remainder of the paper is organized as follows. In Section 2, the CFD model was described and validated against wind-tunnel experimental data. In Section 3, the setup of numerical experiments of different street canyon configurations was given. And the results and discussions of the numerical experiments are then presented in Section 4, and finally the conclusions are given in Section 5.

\section{Model Description and Validation}

In this section, the CFD model used was described and then validated against the wind-tunnel experiments.

2.1. Model Description. As CFD model, the Open Source Field Operation and Manipulation (OpenFOAM) software package version 2.1.1 was used. Further details on OpenFOAM can be found at http://www.openfoam.com/.

In this study, the simpleFoam solver, one stander solver of the OpenFOAM, was used to solve the Reynolds-averaged Navier-Stokes (RANS) equations with the $k-\varepsilon$ turbulence model by using the SIMPLE scheme [24]. The SIMPLE scheme was an iterative method to solve the algebraic system of equations. A termination criterion of $10^{-4}$ was used for all field variables here. And three $k-\varepsilon$ turbulence models, including the standard $k-\varepsilon$ model, the renormalization group 
(RNG) $k-\varepsilon$ model [14], and the realizable $k-\varepsilon$ model [25], were employed and validated against two independent windtunnel experiments of street canyon.

The RANS equations include mass equation, momentum equation, and mass transport equation, which are expressed by the following:

$$
\begin{gathered}
\frac{\partial u_{j}}{\partial x_{j}}=0 \\
\frac{\partial u_{i}}{\partial t}+\frac{\partial}{\partial x_{j}}\left(u_{j} u_{i}\right)-\frac{\partial}{\partial x_{j}}\left[v_{\mathrm{eff}}\left(\frac{\partial u_{i}}{\partial x_{j}}+\frac{\partial u_{j}}{\partial x_{i}}\right)\right]=-\frac{\partial p^{*}}{\partial x_{i}}, \\
\frac{\partial C}{\partial t}+\frac{\partial}{\partial x_{j}}\left(u_{j} C\right)-v_{d} \frac{\partial^{2} C}{\partial x_{k}{ }^{2}}=S
\end{gathered}
$$

where $u_{i}$ is the $i$ th velocity component, $C$ is the pollutant concentration, and $S$ is the source term of the pollutant. In (2), $v_{\text {eff }}$ and $v_{d}$ are the effective kinematic viscosity and diffusion coefficient, which are expressed as follows:

$$
\begin{gathered}
v_{\text {eff }}=v_{o}+v_{t}, \\
v_{d}=\frac{v_{t}}{S_{\mathrm{ct}}},
\end{gathered}
$$

where $v_{o}$ and $v_{t}$ are the molecular viscosity and turbulence viscosity, $S_{\mathrm{ct}}$ is the Schmidt number, and $P^{*}$ is a modified mean kinematic pressure [26].

For every numerical simulation case, the steady state of airflow fields was firstly calculated by using the iterative SIMPLE scheme, and then the numerical results were taken as input for the transient state solution with the pollutant dispersion simulation by using the mass transport equation.

2.2. Two-Dimensional Street Canyon Experiment. The first wind-tunnel experimental dataset used for open validation was got from the wind-tunnel study by Meroney et al. [9] and Rafailidis [27], which was well documented and available on the Internet: http://www2.dmu.dk/atmosphericenvironment/trapos/cfd-wg.htm. It consisted of six buildings and five parallel street canyons, in a two-dimensional experimental setup at a scale of 1:500. The buildings were square, whose width and height were both $60 \mathrm{~mm}$. The wind was perpendicular to the axis of the street, and the line source of tracer was placed on the ground level in the third downstream canyon, where the zone for validation was. The measurements were got from specific points on the walls and roof of the third downstream canyon, which were indicated in Figure 1(d).

Since the wind-tunnel experiment was performed with a scale model, the computational domain was also scaled down to match, which was $600 \mathrm{~mm} \times 480 \mathrm{~mm}$ for the $x$ direction and $z$-direction with a finer grid spacing $(2 \mathrm{~mm})$ for the third street canyon. The concentration measurements of the wind-tunnel experiment were given in a normalized form as follows:

$$
K=\frac{c U L H}{Q}
$$

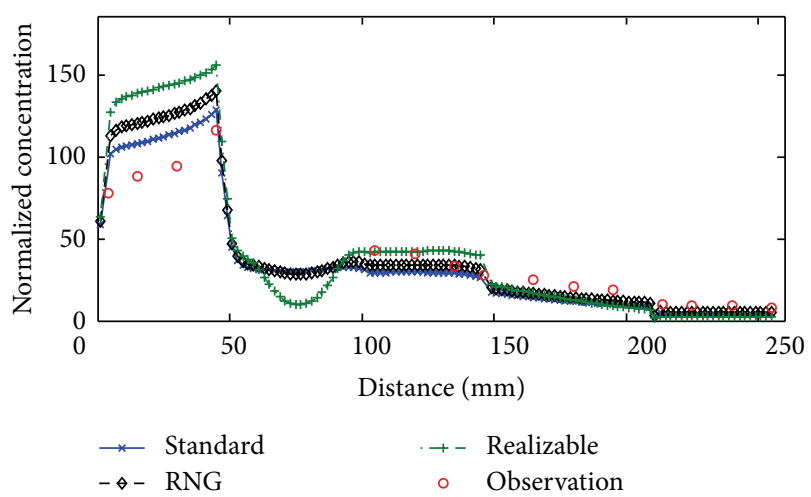

FIGURE 2: Comparison of measured and simulated concentrations along the sampling route indicated in Figure 1(d).

with $K$ the normalized concentration, $c$ the measured concentration, $U$ the refer velocity $(5 \mathrm{~m} / \mathrm{s}), H$ the building height, $L$ the source length (the $y$-direction computational dimension), and $Q$ the source strength.

The concentration distributions and velocity streamline patterns of different turbulence models are given in Figure 1. The streamline patterns are all almost the same; that is, a clockwise vortex is formed right in the center of the street canyon, which drives the tracers to concentrate on the leeward side of the upstream building; and some significant differences of concentration distributions could be found, which are caused by the different mass diffusion coefficients calculated by the different parameterized schemes of the turbulent kinetic energy $(k)$ and dissipate rate $(\varepsilon)$ of the turbulence models. It is found that the tracers are well mixed when employing the standard $k-\varepsilon$ model, with the highest concentration in the central vortex of the street canyon (Figure 1(a)), while the realizable $k-\varepsilon$ model tends to drive the tracers to transport along the walls and floor, whose central part's concentration is the lowest (Figure 1(c)), and the simulation of RNG $k-\varepsilon$ model seems to be the transition state of the other two models (Figure 1(b)).

In order to better understand the performances of the three turbulence models, the measured and simulated concentrations along the sampling route (Figure $1(\mathrm{~d})$ ) are demonstrated in Figure 2. It is found that all the $k-\varepsilon$ models overestimate the concentrations on the leeward side of the upstream building, and the concentrations on the windward side seem more consistent with the measurements. Among these three turbulence models, the standard $k-\varepsilon$ model is the most accurate one in this two-dimensional experiment.

2.3. Three-Dimensional Street Canyon Experiment. The second wind-tunnel experiment used for OpenFOAM validation was carried out at the Laboratory of Buildings and Environmental Aerodynamics at the University of Karlsruhe, which was a three-dimensional experiment. The test section of the wind-tunnel was $2 \mathrm{~m}$ wide, $1 \mathrm{~m}$ high, and $2 \mathrm{~m}$ long, with an isolated street canyon model exposed to the perpendicular approaching flows. The wind-tunnel model consisted of two parallel buildings of $0.12 \mathrm{~m} \times 0.12 \mathrm{~m} \times 1.2 \mathrm{~m}$ (Height $\times$ Width 


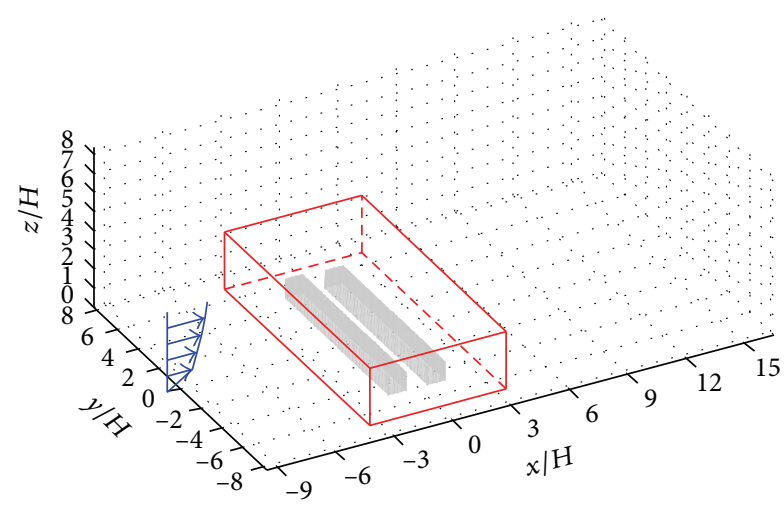

Figure 3: The computational domains of wind-tunnel simulated cases. The finer resolution domain is indicated by the red box inside the computational domain. The origin of coordinates is set right at the center of the street canyon.

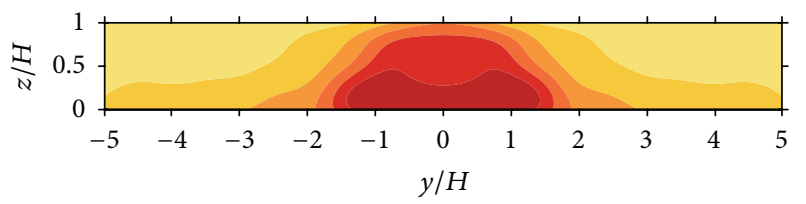

(a) Standard

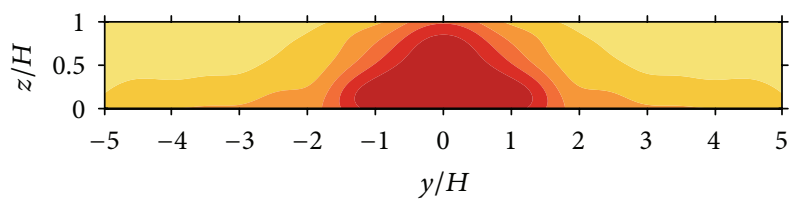

(b) RNG

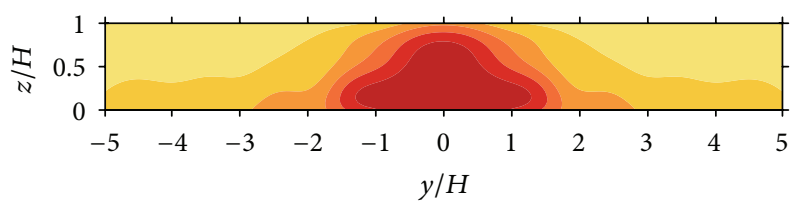

(c) Realizable
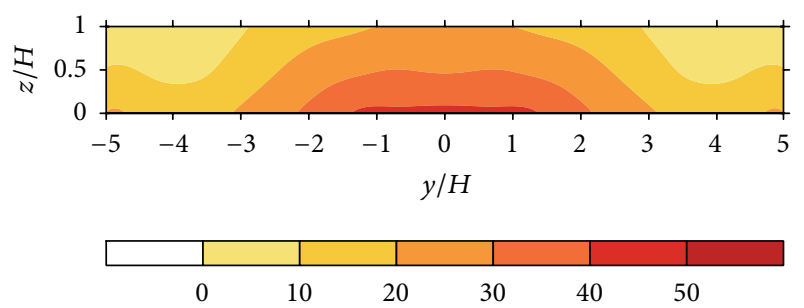

(d) Observation

Figure 4: Normalized concentration distribution on the leeward wall $(x=-0.5 H)$ of the canyon street.

$\times$ Length), which were at a scale of $1: 150$ to the actual street canyon, and the distance between the two parallel buildings was $0.12 \mathrm{~m}$. In the following parts of this paper, the width refers to the width of buildings without special pointing out.

Four tracer gas emitting line sources were embedded at street surface level for simulating the release of traffic

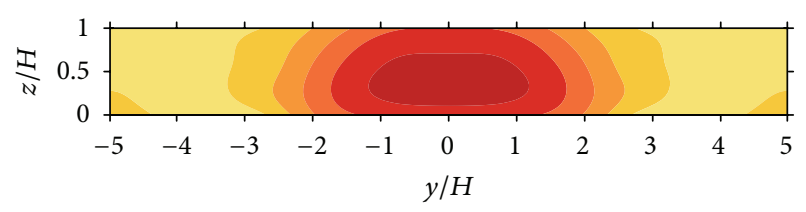

(a) Standard

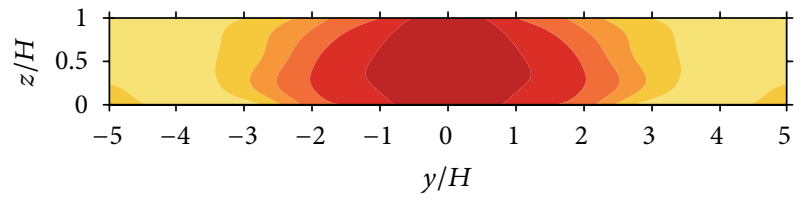

(b) RNG

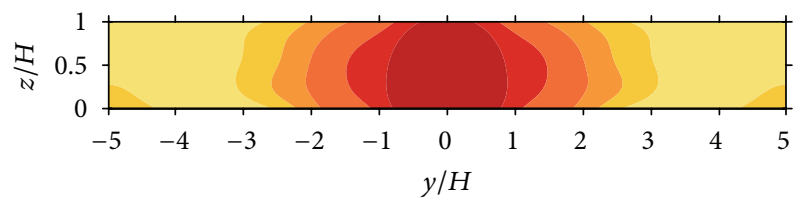

(c) Realizable
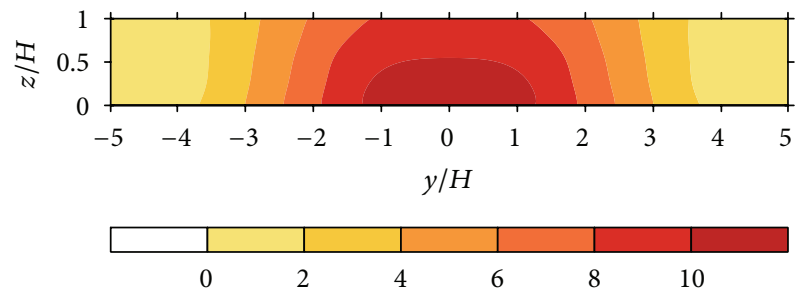

(d) Observation

FIgURE 5: Normalized concentration distribution on the windward wall $(x=0.5 H)$ of the canyon street.

exhausts $[28,29]$. Sulfur hexafluoride $\left(\mathrm{SF}_{6}\right)$ was used as tracer gas, and concentrations were measured at the two canyon walls, normalized by the following equation:

$$
c^{+}=\frac{c U_{H} H}{Q_{L}}
$$

where $c$ is the measured concentration, $H$ is building height, $U_{H}$ is the flow velocity at height $H$ in the undisturbed approaching flow, and $Q_{L}$ is emission rate of line source.

The measurements of normalized concentrations of this three-dimensional wind-tunnel experiment are available on the Internet; details could be found at http://www.ifh.unikarlsruhe.de/science/aerodyn/CODASC.htm.

The three-dimensional numerical experiment used to evaluate OpenFOAM was setup as closely as the actual windtunnel experiment-the cross-sectional dimensions of the computational domain were set equal to the dimensions of the wind tunnel, that is, $1 \mathrm{~m}$ high and $2 \mathrm{~m}$ wide. According to the best practice guidelines of COST Action 732 [30], the upstream distance from the inlet plane to the building was set to $8 H$, and the downstream distance from the outflow plane to the building was set to $15 \mathrm{H}$.

The computational domain was built by using the hexahedral elements, and a finer resolution was set to the region close to the street canyon, indicated by the red box in Figure 3, 


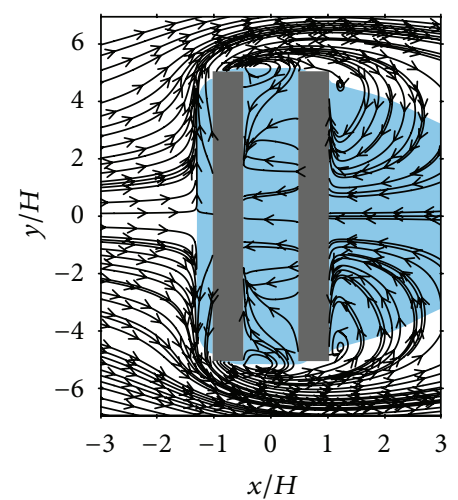

(a) $z=0.1 H$

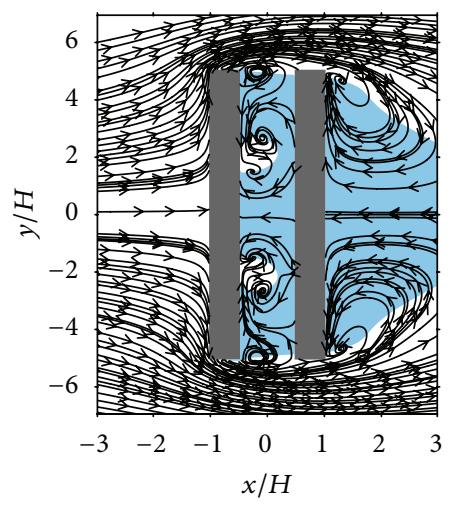

(d) $z=0.5 H$

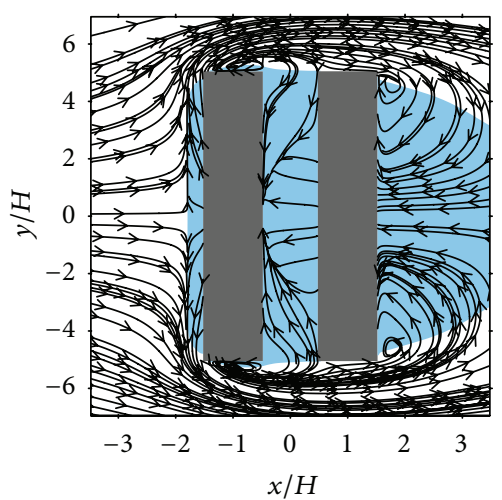

(b) $z=0.1 H$

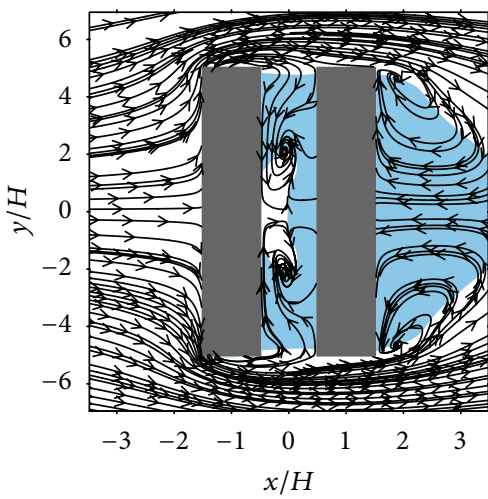

(e) $z=0.5 H$

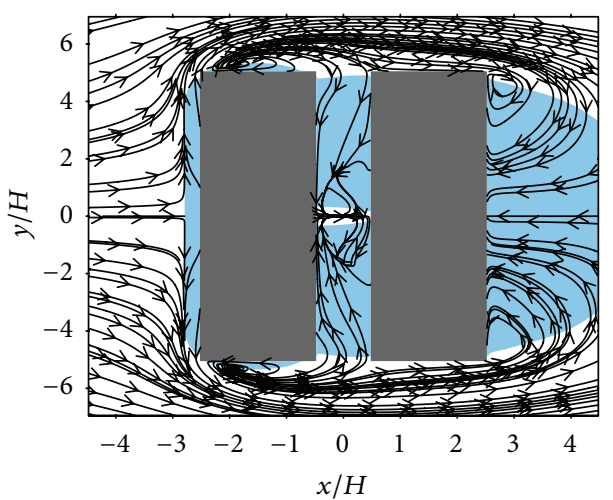

(c) $z=0.1 H$

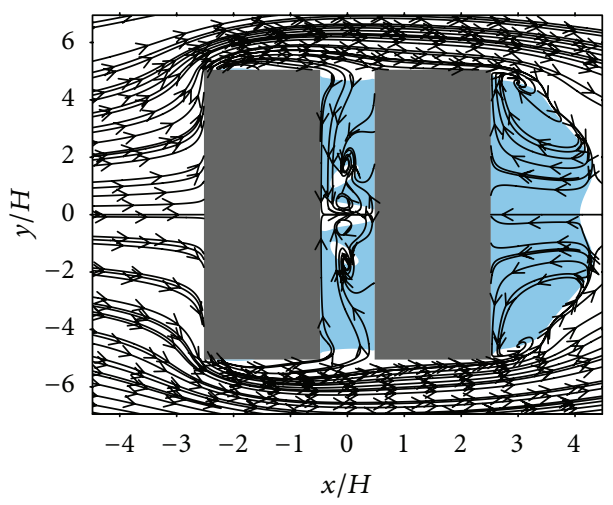

(f) $z=0.5 \mathrm{H}$

FIgURE 6: The streamlines of horizontal section at different height of Experiment 1. (a), (d) Width = 0.5H; (b), (e) Width = H; (c), (f) Width $=2 H$, where the $\mathrm{u}$ component is negative and is indicated by blue shade.

where the distances from the street canyon were smaller than $2 \mathrm{H}$ at the three-dimensional directions. The smaller dimensions of $x$-direction, $y$-direction, and $z$-direction were $1 / 24 H, 1 / 10 H$, and $1 / 24 H$, respectively, and the coarser resolution of the computational domain was all set to $1 / 3 \mathrm{H}$ at the three-dimensional directions.

And the initial inlet wind speed, turbulent kinetic energy, and dissipation rate were all set based on the measurements of wind-tunnel experiments. The Reynolds number of the main flow in the wind-tunnel experiment is 50,000, so as to the replicated numerical experiment. According to the study of Gromke et al. [28], the Schmidt number was set to 0.3 for this wind-tunnel experiment.

Since only the measurements of the normalized concentration of the wind-tunnel experiment are available on the Internet, the three turbulence models are validated against the observed concentration fields. The comparisons of simulated and measured normalized concentrations are given in Figures 4 and 5.

Figure 4 shows the normalized concentrations on the leeward wall $(x=-0.5 H)$ of the street canyon under the perpendicular approaching flow; the simulations are similar to the two-dimensional case, which are overrated by all the three $k-\varepsilon$ models, and the simulations of the standard $k$ $\varepsilon$ model are the most close to the measurements. On the windward side of the street canyon, the distributions of simulated concentration seem to be more in agreement with the measurements (Figure 5).

To better understand the performances of OpenFOAM, three validation metrics are selected to quantify the degree of correlation between experimental data and OpenFOAM simulation of the three-dimensional experiment, which are the fractional bias (FB), the root normalized mean square error (RNMSE), and the Pearson correlation coefficient $(R)$.

The FB is expressed as follows:

$$
\mathrm{FB}=2 \frac{\overline{c_{o}^{+}}-\overline{c_{s}^{+}}}{\overline{c_{o}^{+}}+\overline{c_{s}^{+}}}
$$

where the subscripts $o$ and $s$ refer to observations and simulations. A negative FB illustrates that the observations are lower than the simulations.

The RNMSE is the square root of the normalized mean square error (NMSE) as follows:

$$
\begin{aligned}
& \text { NMSE }=\frac{\overline{\left(c_{o}^{+}-c_{s}^{+}\right)^{2}}}{\overline{c_{o}^{+} \cdot \overline{c_{s}^{+}}}}, \\
& \text {RNMSE }=\sqrt{\text { NMSE }} .
\end{aligned}
$$




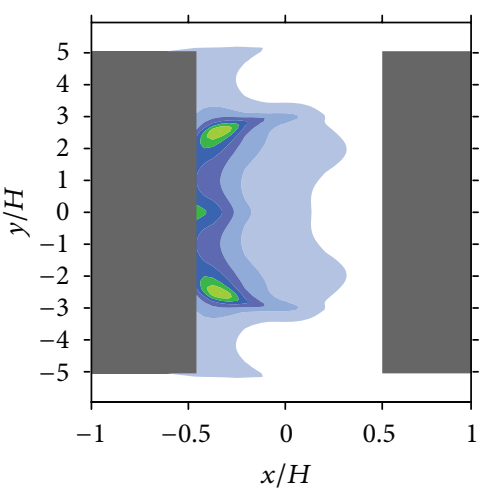

(a) $z=0.1 H$

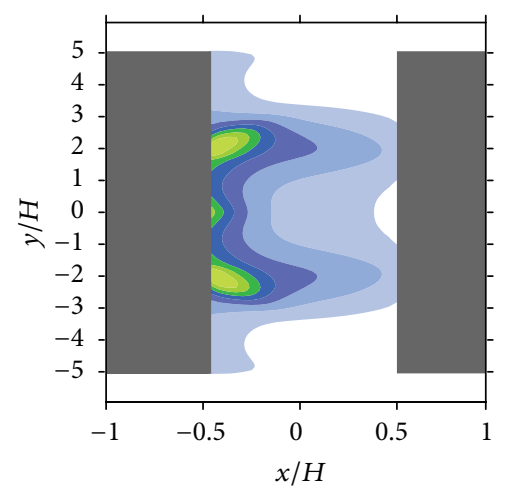

(d) $z=0.5 H$

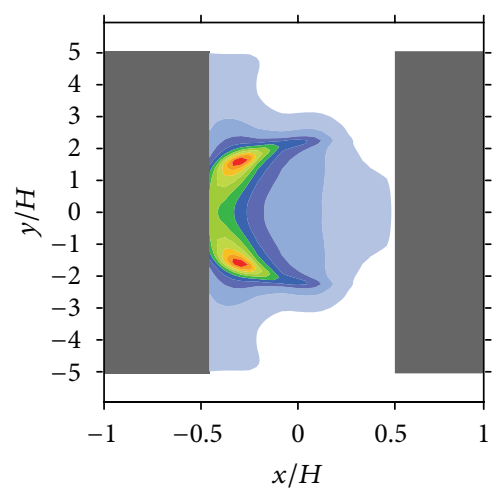

(b) $z=0.1 H$

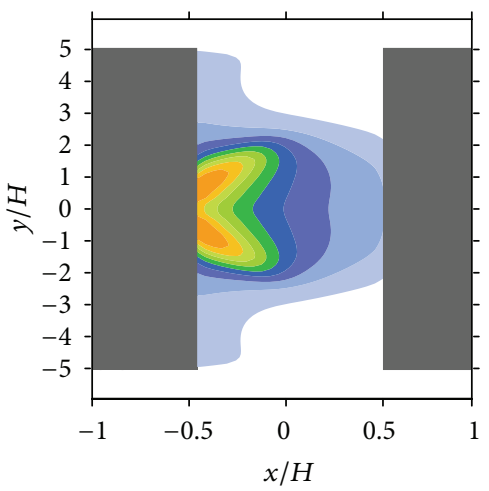

(e) $z=0.5 H$

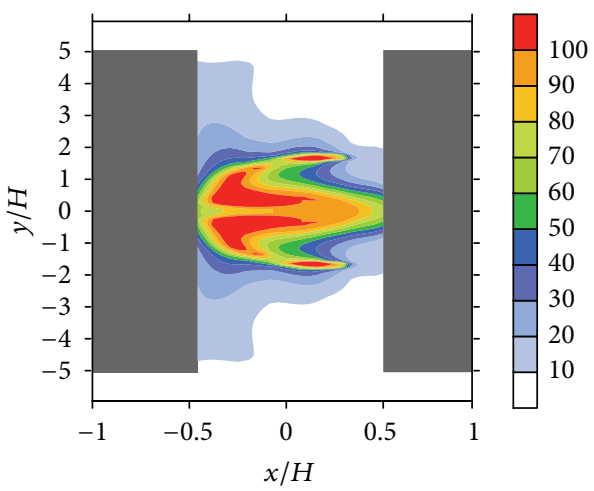

(c) $z=0.1 H$

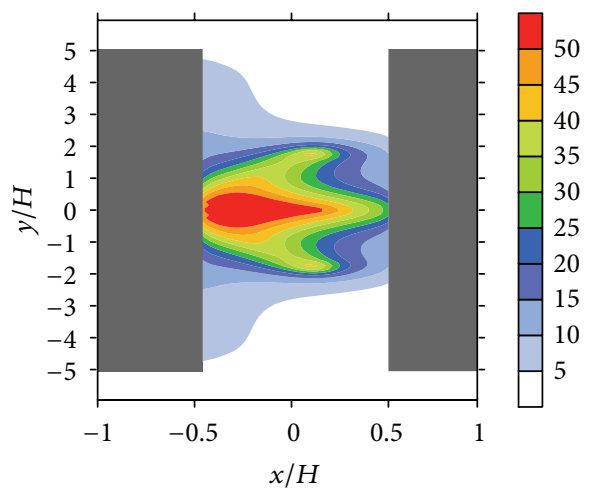

(f) $z=0.5 \mathrm{H}$

FIGURE 7: The normalized concentration of horizontal section at different height of Experiment 1. (a), (d) Width = 0.5H; (b), (e) Width = $H$; (c), (f) Width $=2 H$.

The RNMSE is a direct measure of the difference between simulations and observations.

The $R$ is a measure of the correlation between simulations and observations, which is expressed by the following:

$$
R=\frac{\overline{c_{o}^{+}-\overline{c_{o}^{+}}}}{\sigma_{o}} \frac{c_{p}^{+}-\overline{c_{p}^{+}}}{\sigma_{p}},
$$

where $\sigma_{o}$ and $\sigma_{p}$ are the standard deviations on observed and simulated mean normalized concentrations.

To check whether the model performance is acceptable or not, some studies [29] gave the following limit values for the metrics mentioned above: $-0.3<\mathrm{FB}<0.3$, NMSE $<$ 1.5 , and $R>0.8$. The results of these validation metrics are summarized in Table 1.

It is found that all the values of validation metrics fall inside the accepted range. Specifically, FB reveals small overestimation of simulations, which is consistent with the interpretation of Figure 4. Among these three turbulence models, the standard $k-\varepsilon$ model has the smallest absolute value of FB and RNMSE and the largest correlation coefficient ( $R=0.912)$, which is the most accurate turbulence model for this numerical experiment.

From this preliminary validation, it is concluded that when the wind was perpendicular to the axis of the street, the $k-\varepsilon$ models seemed to overrate the concentration on the leeward side of street canyon (Figures 2 and 4). Despite this discrepancy, the wind flow pattern and concentration distribution within the street canyon were reasonable simulated by the OpenFOAM by using these turbulence models, and the standard $k-\varepsilon$ model was the most accurate, which would be used to study the flow and dispersion patterns within different configurations of street canyon.

\section{Numerical Experimental Setup of Different Configurations}

Three different kinds of street canyon configuration under the perpendicular approaching flow were considered in this study, which were summarized in Table 2.

In Experiment 1, three different values of width were set to examine the effect of the width on the dispersion of pollutant within the street canyon. And Experiment 2 focused on the effect of the height of buildings. Finally, two kinds of asymmetrical street canyon were designed in Experiment 3. While varying a particular parameter (width or height), all other dependent parameters are kept constant.

It is noted that all Part B of the three numerical experiments (Table 2) is the same three-dimensional experiment used for the model validation under the perpendicular approaching flow mentioned above, and all the computational domains of other experiments are set similar to the 


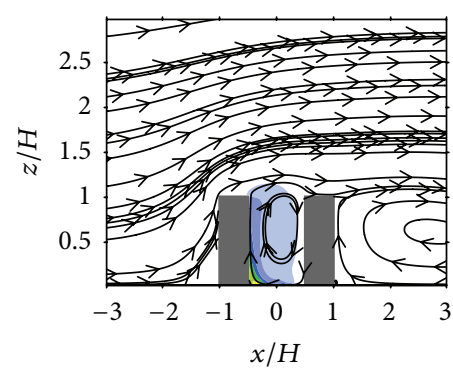

(a) $y=0 H$

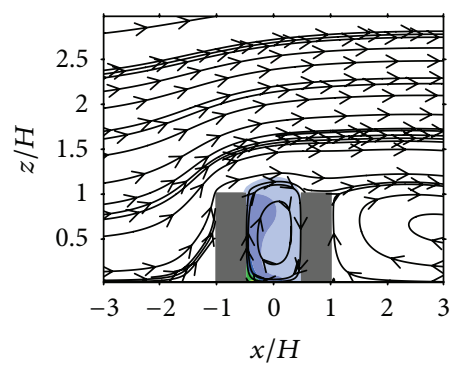

(d) $y=1.5 H$

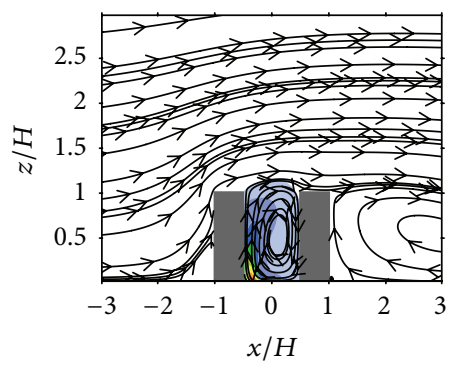

515253545556575

(g) $y=2.5 H$

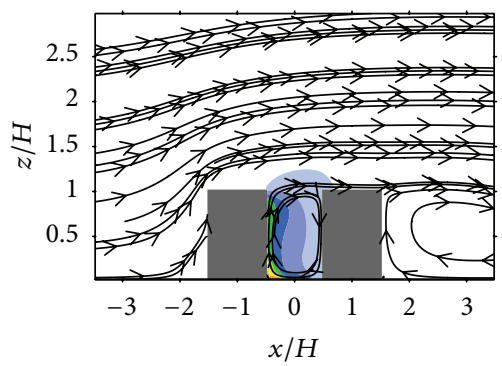

(b) $y=0 H$

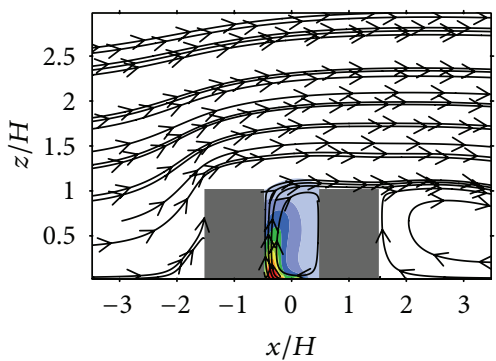

(e) $y=1.5 H$

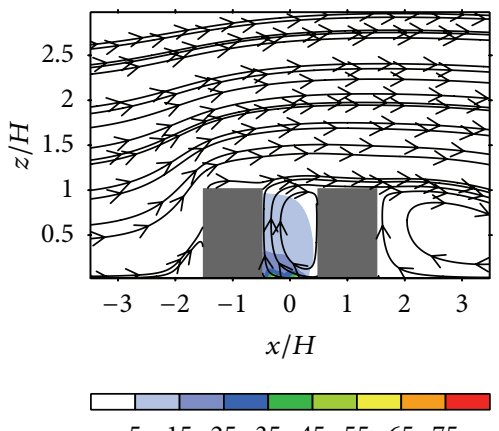

(h) $y=2.5 H$

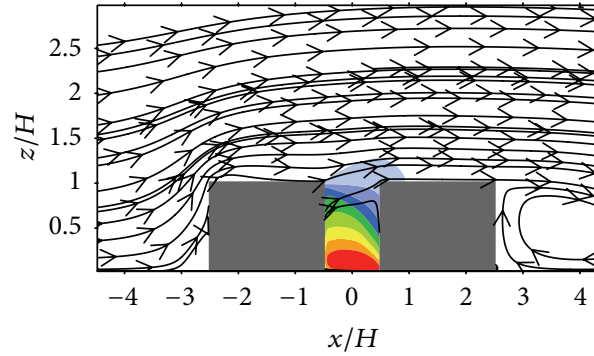

(c) $y=0 H$

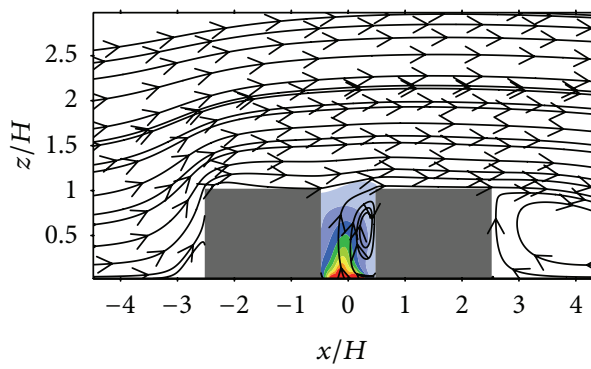

(f) $y=1.5 H$

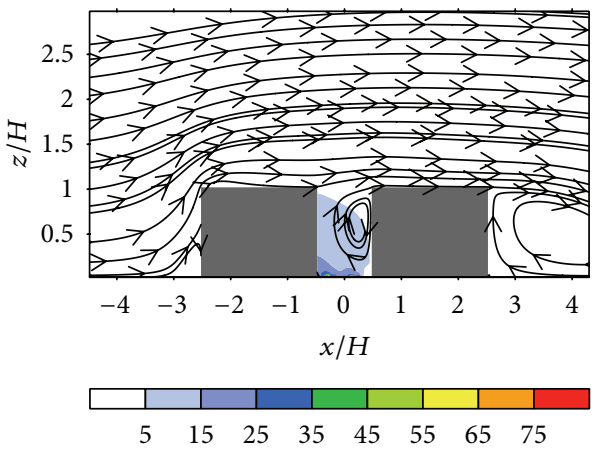

(i) $y=2.5 H$

FIGURE 8: The streamlines and normalized concentration of vertical plane of Experiment 1. (a), (d), and (g) Width = 0.5H; (b), (e), and (h) Width $=H$; (c), (f), and (i) Width $=2 H$.

TABLE 1: Validation metrics for the model performance. Target values and accepted range are also illustrated here.

\begin{tabular}{lccccc}
\hline Metric & Aim & Range & Standard $k-\varepsilon$ & RNG $k-\varepsilon$ & -0.036 \\
FB & 0 & $|-0.3,0.3|$ & -0.017 & -0.105 & 0.602 \\
RNMSE & 0 & $<\sqrt{1.5}$ & 0.557 & 0.662 & 0.659 \\
$R$ & 1 & $>0.8$ & 0.912 & 0.886 & 0.877 \\
\hline
\end{tabular}

three-dimensional case (Figure 3), namely, finer resolution close to the street canyon; the upstream and downstream distances are $8 \mathrm{H}, 15 \mathrm{H}$, and so forth. And the setup of pollutant sources is also identical to the three-dimensional validation case.

\section{Results and Discussion}

4.1. Effect of the Width of Buildings. As listed in Table 2, the street canyons formed by buildings with three different widths $(0.5 H, 1 H$, and $2 H)$ are examined here. The streamlines of horizontal section at different heights are given in Figure 6, where the u component of wind vector opposite to the inlet flow is indicated by the blue shade. It is noted that when the free stream impacts against a building perpendicularly, the stream would diverge into two substreams in front of the buildings and flow across the building.

At the lower level $(z=0.1 H)$ horizontal plane, two lateral vortexes are formed at the lateral (shorter) side of the upstream building. It is interesting that the distances from the center of lateral vortexes to the windward side of the upstream buildings along the $x$-direction are nearly 


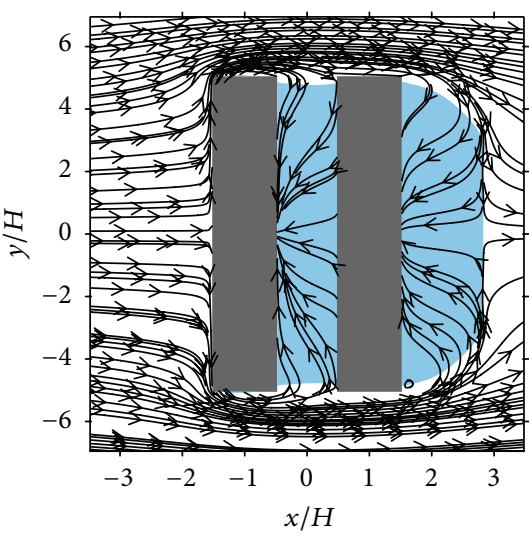

(a) $z=0.1 H$

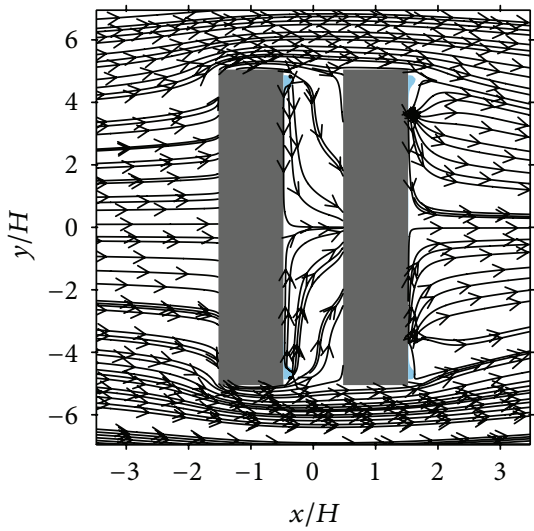

(d) $z=0.35 H$

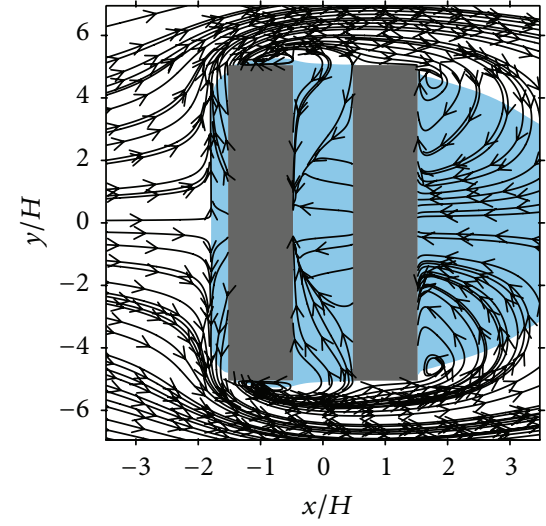

(b) $z=0.1 H$

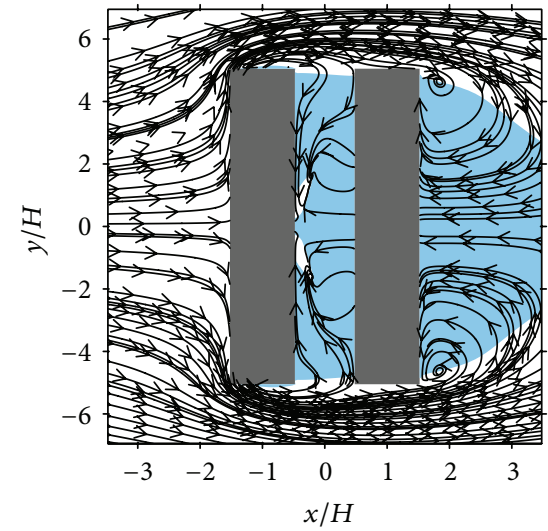

(e) $z=0.35 H$

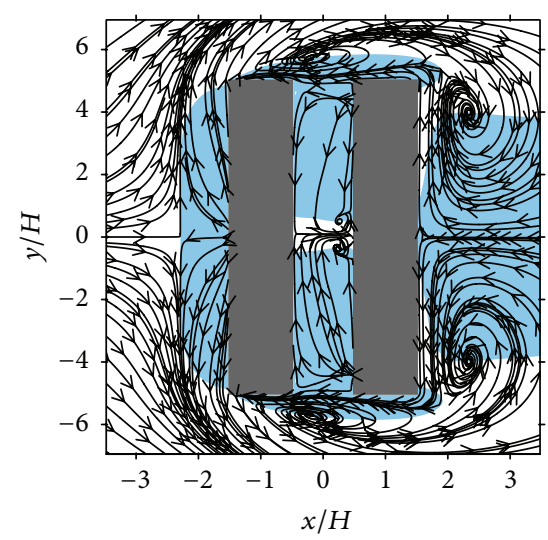

(c) $z=0.1 H$

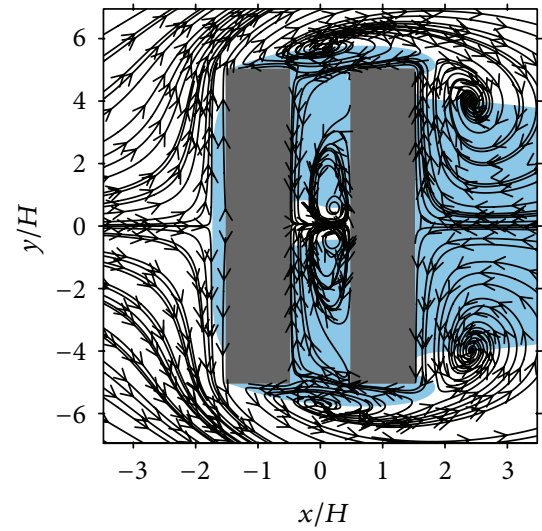

(f) $z=0.35 H$

FIGURE 9: The streamlines of horizontal section at different height of experiment. (a), (d) Height = 0.5H; (b), (e) Height = H; (c), (f) Height $=2 \mathrm{H}$, where the $\mathrm{u}$ component is negative and is indicated by blue shade.

TABLE 2: Summary of three kinds of street canyon configurations. $H$ is the height of building in the wind-tunnel experiment; namely, $H=$ $0.12 \mathrm{~m} . H_{u}$ and $H_{d}$ are the heights of the upstream and downstream building.

\begin{tabular}{lcccc}
\hline Exp. number & Variable of buildings & Part A & Part B & Part C \\
\hline 1 & Width & $0.5 H$ & $H$ & $2 H$ \\
2 & Height & $0.5 H$ & $H$ & $2 H$ \\
3 & $H_{u} / H_{d}$ & 0.5 & 1 & 2 \\
\hline
\end{tabular}

constant among these three cases (Figures 6(a)-6(c)), which is about $0.8 \mathrm{H}$. Therefore, as the width increases, the relative positions of the lateral vortexes shift from the corner of the upstream buildings to the middle along the lateral sides. And the flow pattern within the street canyon is driven by the lateral vortexes, so the flow pattern is different as the width increases. When the width $=0.5 \mathrm{H}$, the substreams driven by the lateral vortexes would impact against the leeward side of the upstream buildings and produce two stagnation points located at $x=-0.5 \mathrm{H}$ and $y= \pm 2.4 \mathrm{H}$ (Figure 6(a)); when the width increases to $1 H$, the positions of the stagnation points are more close to the middle of the street canyon (Figure 6(b)), which are $x=-0.5 H$ and $y= \pm 1.5 H$; however, when the width $=2 \mathrm{H}$, it is another flow pattern formed within the street canyon: two substreams converge together in the middle of street canyon and flow toward the downstream building (Figure 6(c)).

At the height of $0.5 H$ (Figures 6(d)-6(f)), some vortexes are formed inside the street canyon, whose locations are consistent with the positions of stagnation points or convergent zones on the lower level $(z=0.1 H)$.

From the flow patterns given in Figure 6, it is obvious that with the increasing of width, the stagnation points of the substreams move more close to the middle of the leeward side of the upstream building; when the stagnation points are close enough, the substreams would coverage together in the middle of the street canyon. And the distributions of pollutant concentration are consistent with these flow patterns; as the width increases, the pollutants concentrate more in the middle of street canyon along the leeward side of upstream building (Figure 7 ). It is found that the $2 \mathrm{H}$ width street canyon is the most polluted configuration under the perpendicular approaching flow.

The vertical distributions of flow field and pollutant concentration are given in Figure 8. The patterns of flow and pollutants of the $0.5 \mathrm{H}$ width configuration are similar to the patterns of $1 H$ width street canyon; both have a clockwise 


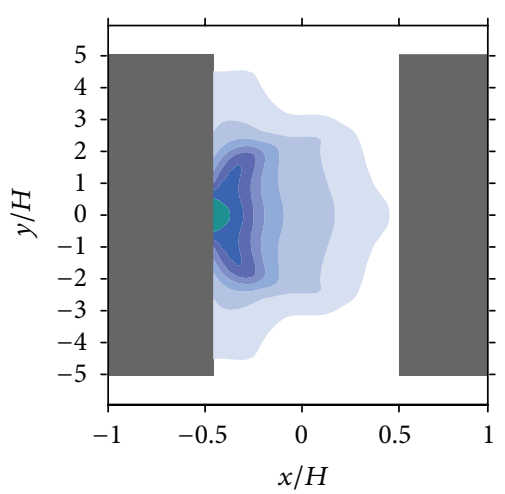

(a) $z=0.1 H$

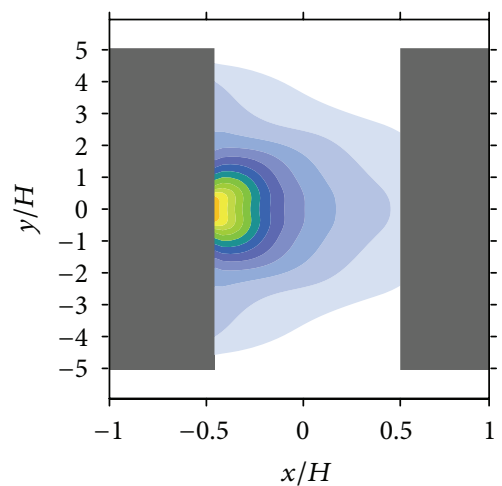

(d) $z=0.35 H$

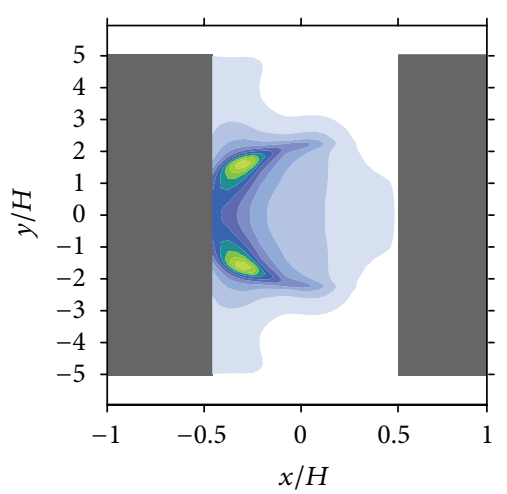

(b) $z=0.1 H$

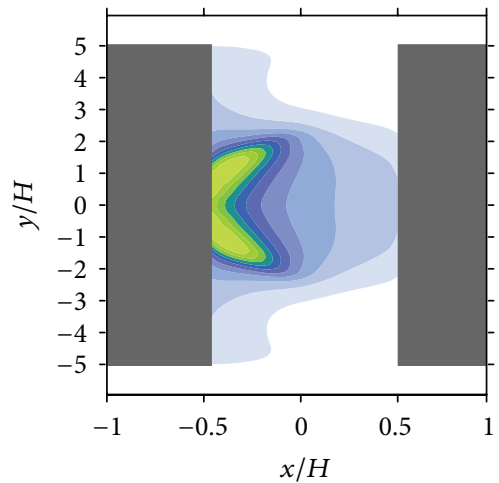

(e) $z=0.35 H$

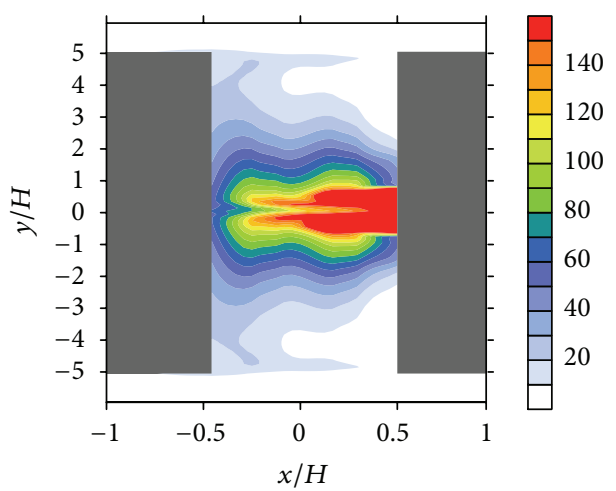

(c) $z=0.1 H$

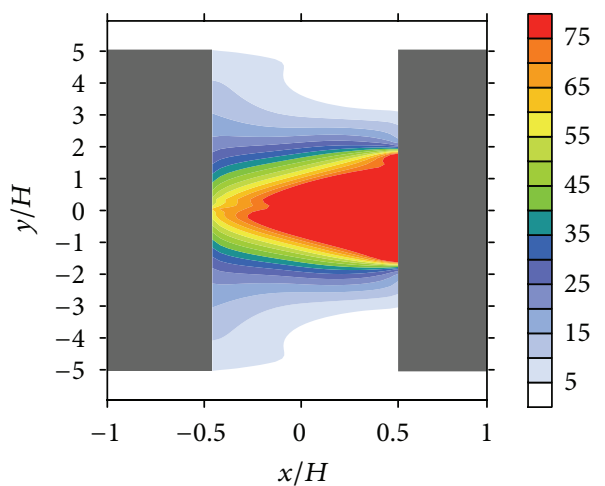

(f) $z=0.35 H$

FIGURE 10: The normalized concentration of horizontal section at different height of Experiment 2. (a), (d) Height = 0.5H; (b), (e) Height = H; (c), (f) Height $=2 H$.

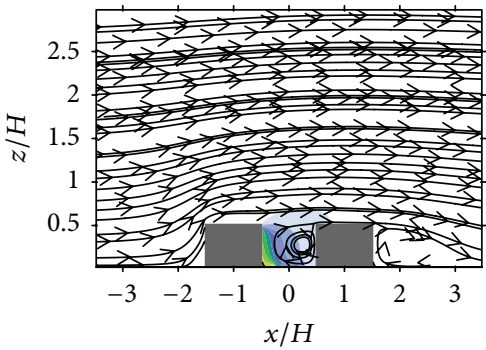

(a) $y=0 H$

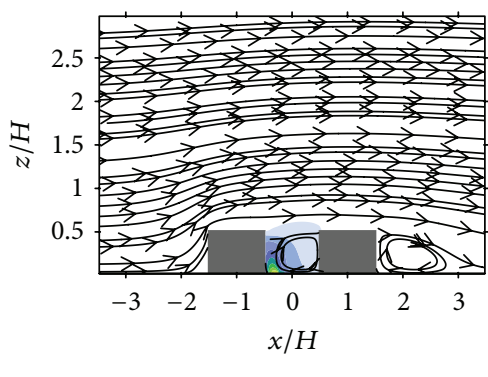

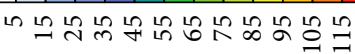

(d) $y=1 H$

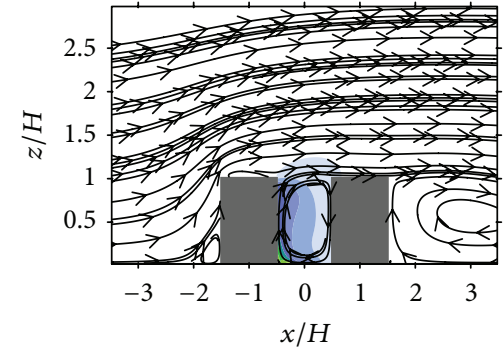

(b) $y=0 H$
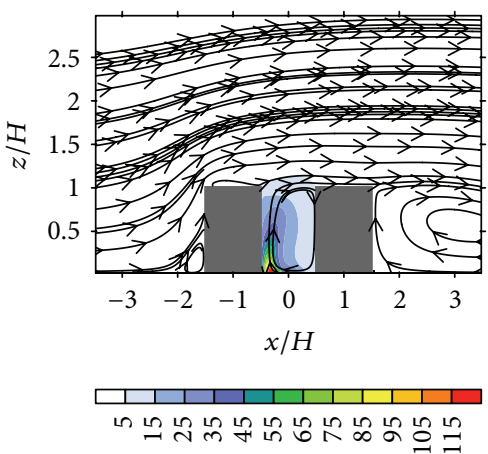

(e) $y=1 H$

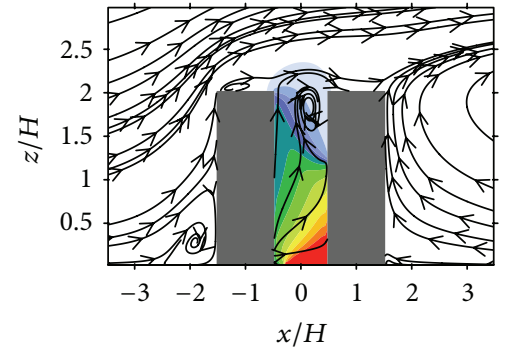

(c) $y=0 H$
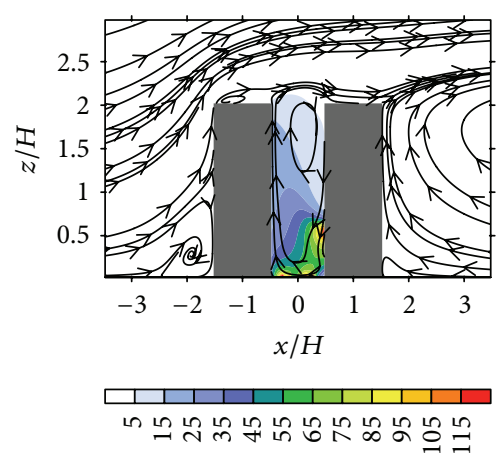

(f) $y=1 H$

FigURE 11: The streamlines and normalized concentration of vertical plane of Experiment 2. (a), (d) Height = 0.5H; (b), (e) Height = H; (c), (f) Height $=2 H$. 


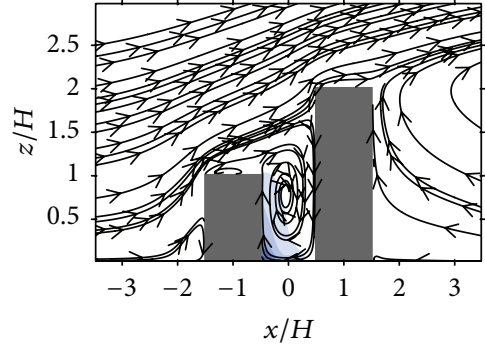

(a) $y=0 H$

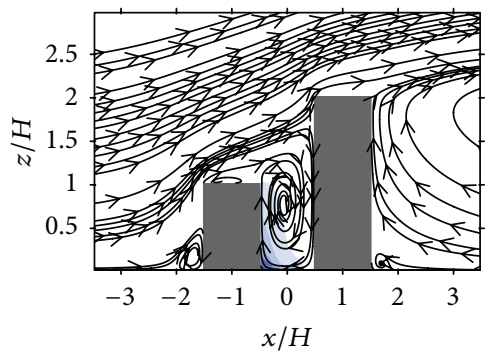

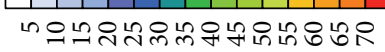

(d) $y=1.5 \mathrm{H}$

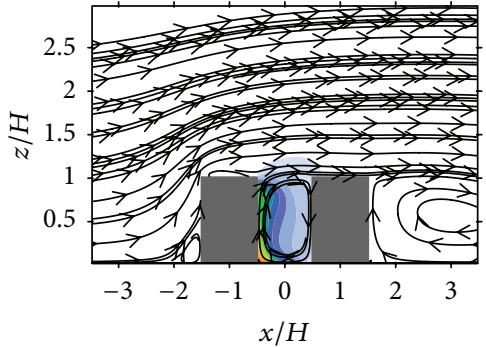

(b) $y=0 H$

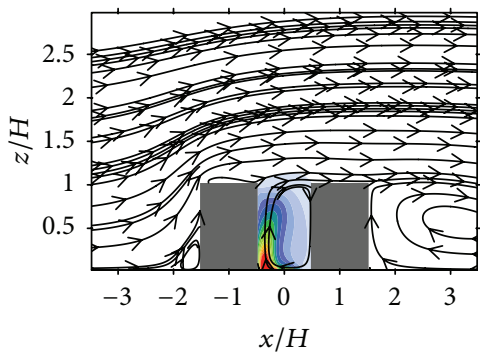

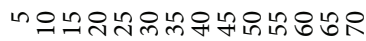

(e) $y=1.5 H$

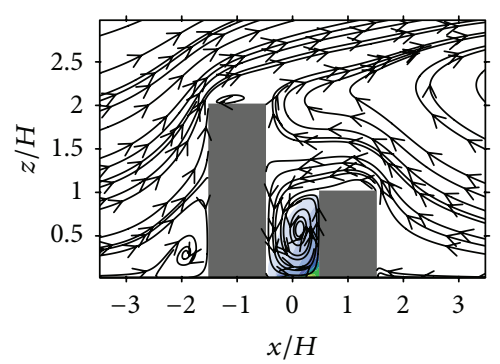

(c) $y=0 H$

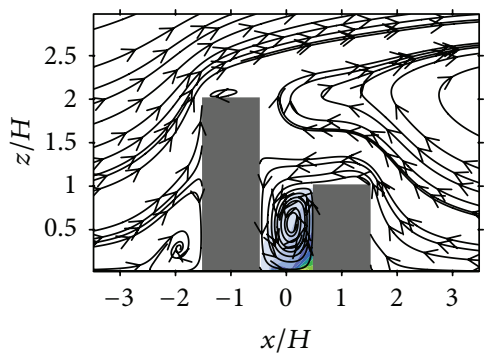

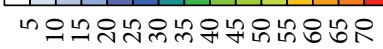

(f) $y=1.5 H$

FIGURE 12: The streamlines and normalized concentration of vertical plane of Experiment 3. (a), (d) $H_{u} / H_{d}=0.5 ;(\mathrm{b}),(\mathrm{e}) H_{u} / H_{d}=1 ;$ (c), (f) $H_{u} / H_{d}=2$.

vortex right in the middle of the street canyon no matter where the vertical section is made $(y=0 H, y=1.5 \mathrm{H}$, and $y=2.5 H$ ), and the vortex could drive the pollutants transport along the leeward side of the upstream building. For the $2 H$ with street canyon, there is no vortex formed in the vertical plane of $y=0 H$, and the pollutants are trapped near the ground with higher concentrations than the other cases. When the vertical section moves a little laterally $(y=1.5 \mathrm{H}$ or $2.5 \mathrm{H}$ ), a weaker vortex could be found near the downstream building. The flow field of $2 \mathrm{H}$ width configuration is not conducive to the diffusion of pollutant released from the ground.

In short, it is found that the change of width of building alters the flow fields and pollution within the street canyon significantly; the longer the width, the heavier the pollution would happen within the street canyon at the pedestrian level.

4.2. Effect of the Height of Buildings. The symmetric street canyon with three different heights $(0.5 H, 1 H$, and $2 H)$ is studied in this study, whose horizontal flow fields are showed in Figure 9. It is found that the variation of height of buildings would dramatically change the flow patterns in the street canyons.

First of all, the strength and positions of the lateral vortexes are different, corresponding to different building height. When the height $=0.5 \mathrm{H}$, the lateral vortexes are too weak to be found at the horizontal planes (Figures 9(a) and $9(\mathrm{~d})$ ). While the lateral vortexes of the tallest configuration (height $=2 \mathrm{H}$ ) are so strong that the lateral sides of the whole canyon street (including both the upstream and downstream buildings) are under their controls (Figures 9(c) and 9(f)).
It is found that the taller street canyon is accompanied with stronger lateral vortexes at the lower height, whose positions are more close to the downstream building.

Secondly, the flow patterns inside the street canyons are also different. At the lower level $(z=0.1 H)$ of $0.5 H$ height street canyon, the substreams could flow across the upstream building and impact against the leeward side of the upstream building (Figure 9(a)), with only one common stagnation point located at the middle of leeward wall $(x=-0.5 \mathrm{H}$ and $y=0 H)$. In contrast, there are two stagnation points located at the leeward side of the $1 H$ height upstream building as described in the previous section. However, corresponding to the difference of the lateral vortexes, the flow pattern of the $2 \mathrm{H}$ height configuration is also dramatically different from the others: a strong convergent zone throughout the street canyon with positive $\mathrm{u}$ wind component is formed in the center of street canyon.

In addition, at the height of $0.35 \mathrm{H}$, the flow patterns of the two taller configurations are both consistent with the wind fields at the lower level $(z=0.1 H)$; while the patterns of $0.5 \mathrm{H}$ height street canyon are totally different from the lower parts. It is because the height $0.35 \mathrm{H}$ is close to the roof level of the $0.5 \mathrm{H}$ height street canyon; hence the air flows within the street canyon are affected by the free stream above the roof level, which are toward the downstream building (Figure 9(d)).

Under these flow fields, the distributions of pollutant are also quite different (Figure 10). The extreme high concentration pattern is found within the tallest street canyon, with the peak value located at the windward side of downstream building (Figures 10(c) and 10(f)), which is quite different 


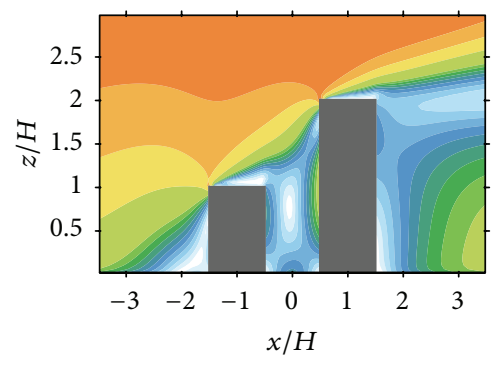

(a) $y=0 H$
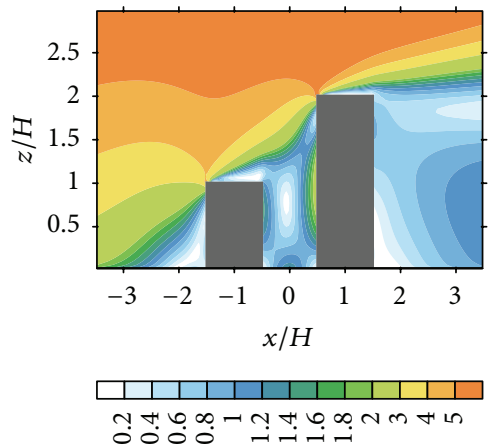

(d) $y=1.5 H$

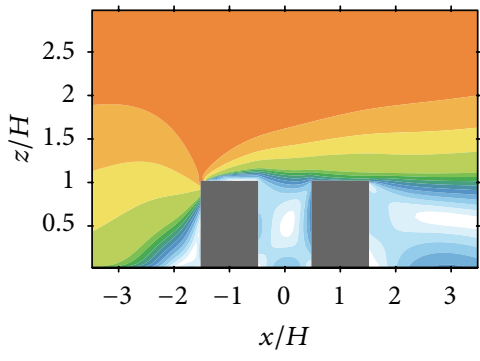

(b) $y=0 H$

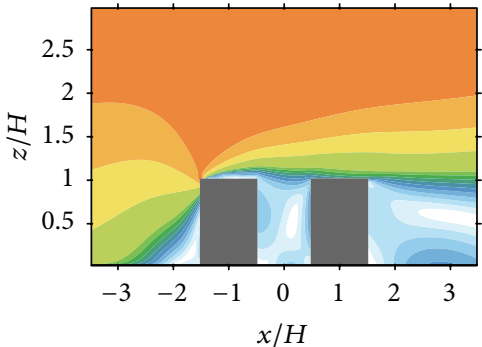

ñ.

(e) $y=1.5 \mathrm{H}$

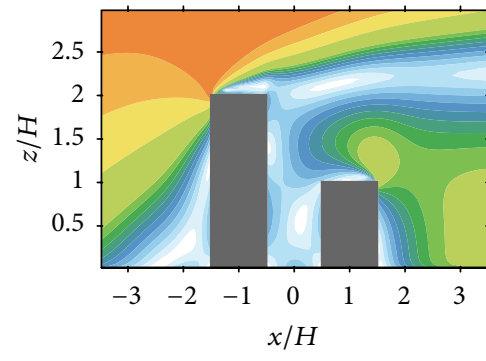

(c) $y=0 H$

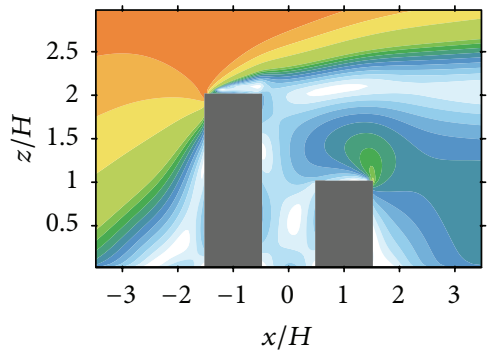

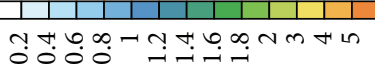

(f) $y=1.5 H$

FIGURE 13: The distribution of wind speed (m/s) of vertical plane of Experiment 3. (a), (d) $H_{u} / H_{d}=0.5$; (b), (e) $H_{u} / H_{d}=1 ;$ (c), (f) $H_{u} / H_{d}=2$.

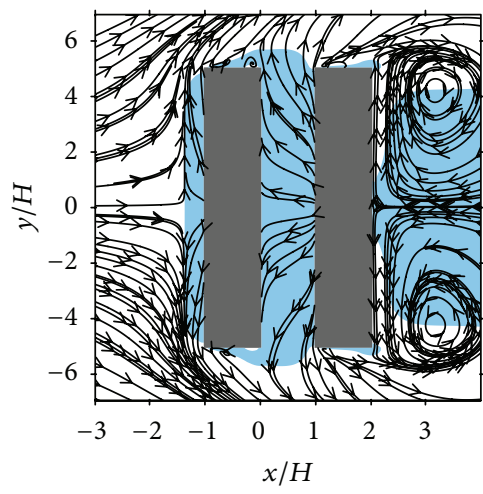

(a) $z=0.1 H$

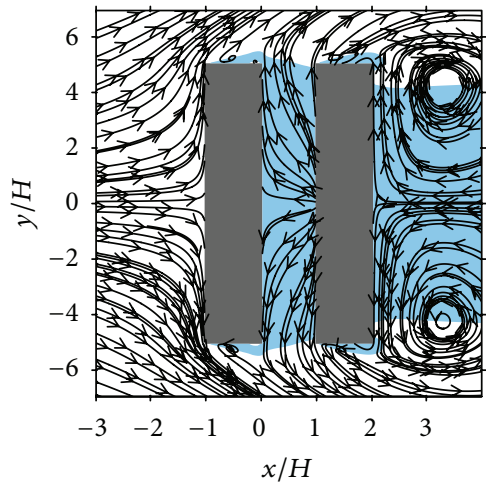

(d) $z=0.35 H$

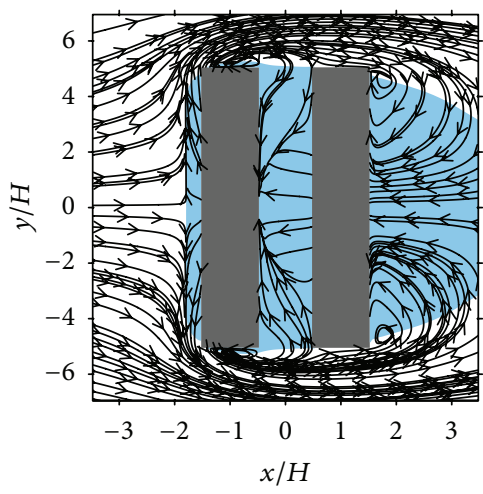

(b) $z=0.1 H$

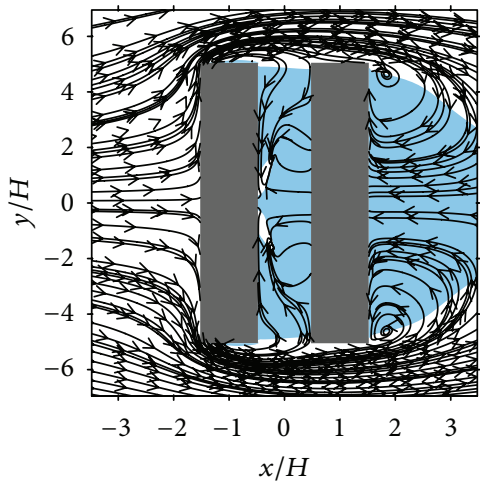

(e) $z=0.35 H$

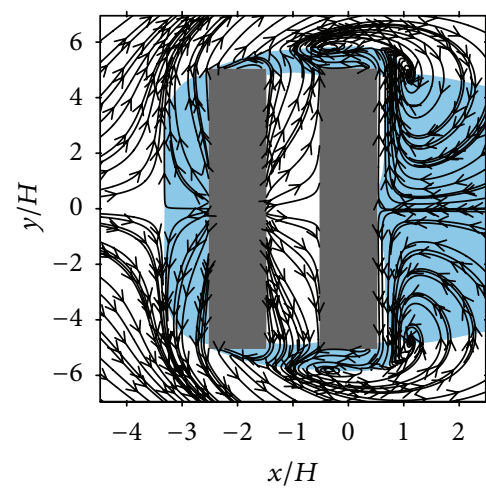

(c) $z=0.1 H$

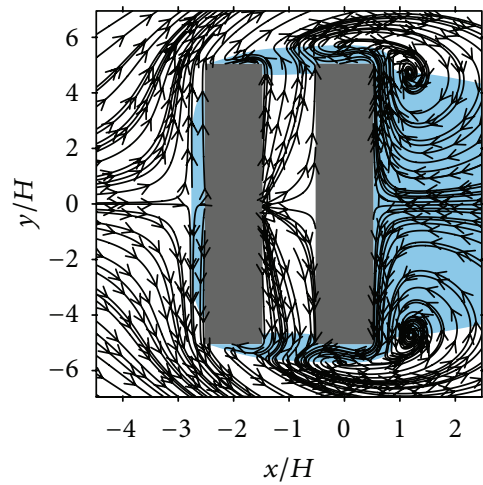

(f) $z=0.35 \mathrm{H}$

Figure 14: The streamlines of horizontal section at different height of Experiment 3. (a), (d) $H_{u} / H_{d}=0.5$; (b), (e) $H_{u} / H_{d}=1$; (c), (f) $H_{u} / H_{d}=2$, where the $\mathrm{u}$ component is negative and is indicated by blue shade. 


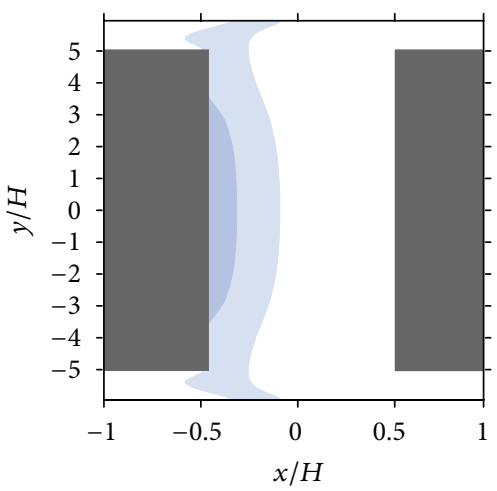

(a) $z=0.1 H$

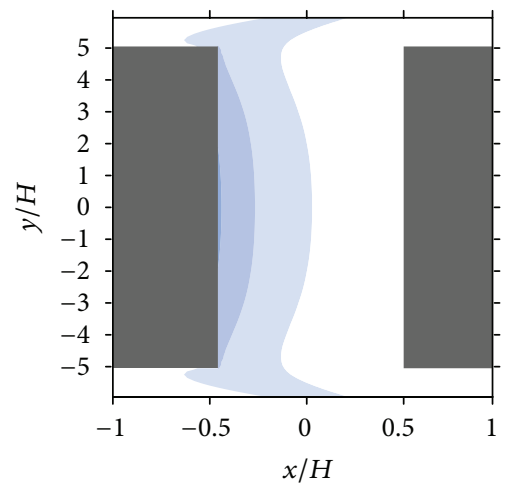

(d) $z=0.35 H$

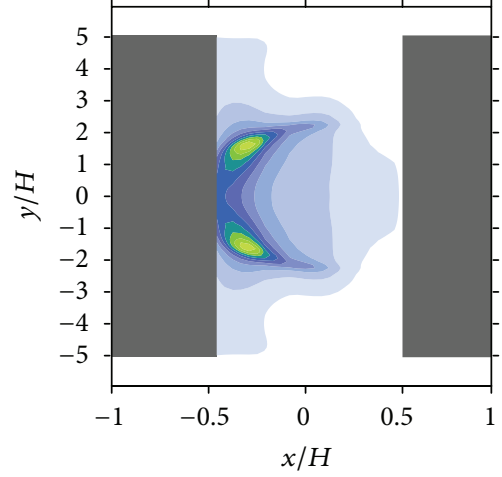

(b) $z=0.1 H$

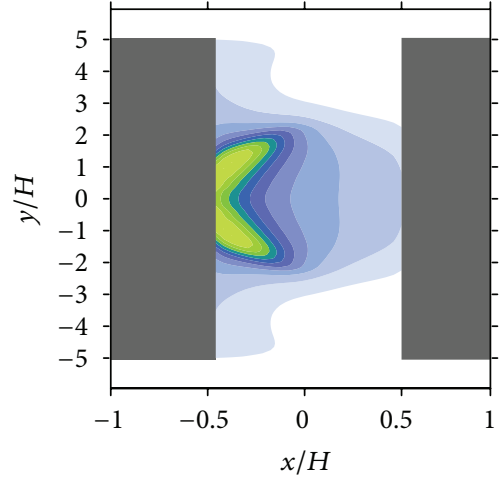

(e) $z=0.35 H$

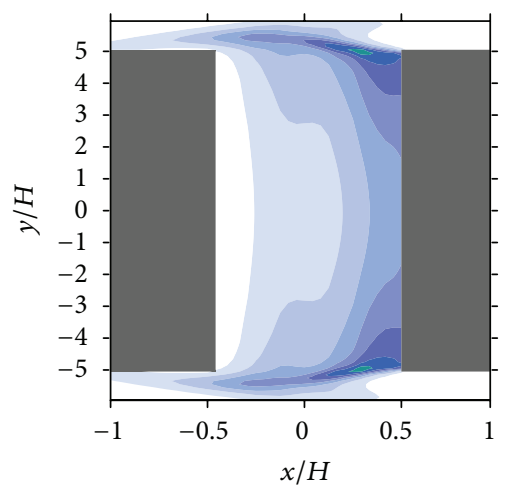

(c) $z=0.1 H$

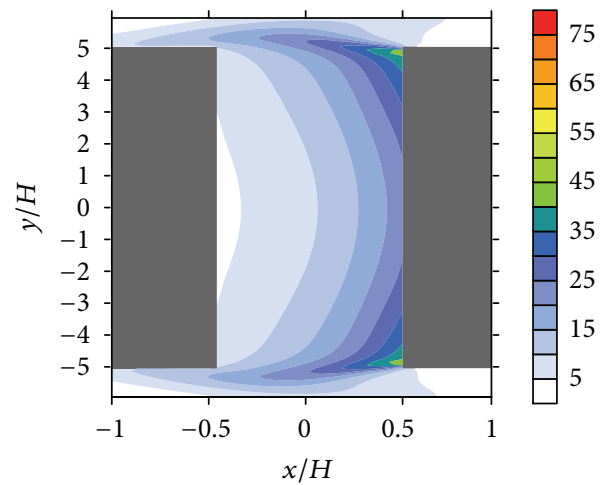

(f) $z=0.35 H$

Figure 15: The normalized concentration of horizontal section at different height of Experiment 3. (a), (d) $H_{u} / H_{d}=0.5$; (b), (e) $H_{u} / H_{d}=1$; (c), (f) $H_{u} / H_{d}=2$.

to other cases. This phenomenon can be explained by the wind fields illustrated in Figures 9 and 11 -the buildings are so tall that vortex in the vertical section is detached from the ground, and the convergent zone in the horizontal plane drives the pollutant to accumulate at the street canyon's center and the windward side of the downstream building.

However, in the other two shorter street canyons $(0.5 \mathrm{H}$ and $1 H$ ), the peak values of concentration are both located at the leeward side of upstream building: one is located right in the middle of leeward side of the upstream building (Figures 10 (a) and 10(d)), while the other splits into two centers located at the positions of $x=-0.4 \mathrm{H}$ and $y= \pm 1.5 \mathrm{H}$ at the horizontal plane (Figures 10(b) and 10(e)). And the vertical flow patterns of these two cases are similar-a clockwise rotated vortex occupies the whole street canyon (Figures 10(a)-10(b) and Figures 11(e)-11(f)).

After comparing the flow and dispersion patterns, it is obvious that, with the increasing of the height of street canyon, the air quality within the street canyon deteriorates.

4.3. Effect of the Step-Up and Step-Down Configurations. The configurations described and discussed above are all symmetric; in this part, the effect of the asymmetric configurations would be examined.
The vertical patterns of concentration and streamlines are demonstrated in Figure 12, and the corresponding wind speeds are given in Figure 13. In comparison with the symmetric street canyon (Figures 12(b) and 13(b)), the heightened height of downstream building would strengthen the vertical vortex within the step-up street canyon (Figures 12(a) and 13(a)). This phenomenon is caused by the blockage of flow in front of the higher downstream building, which enhances the strength of the downdraft flow near the windward side of downstream building (Figures 13(a) and 13(d)), and then the lower parts of the street canyon are occupied by the divergent flows of the strong downdraft flow (Figures 14(a) and 14(d)). Under these divergent flow fields, it is effective to dilute and remove the pollutants within the street canyon (Figures 15(a) and $15(\mathrm{~d}))$.

And for the case of the step-down configuration, as the upstream building heightens, a large recirculating clockwise vortex is formed at the leeward region of upstream building and drives a counterclockwise vortex formed in the vertical plane of the street canyon (Figures 12(c) and 12(f)). The same as the step-up configuration, the lower part of the step-down street canyon is also occupied by the divergent flows (Figures 14(c) and 14(f)), but in the opposite direction; and it is also good for the pollutants transport outside the street canyon under these flow fields. 
In general, in comparison with the symmetric case (Figures 12(b) and 15(b)), although one of the buildings is taller either in the step-up or the step-down configuration, the air quality of the asymmetric cases is better, even better than the $0.5 \mathrm{H}$ height symmetric street canyon case (Figures 11(a) and $11(\mathrm{~d}))$. It is found that the nonuniform configurations provide better ventilation.

\section{Conclusions}

In this study, we first validated OpenFOAM against two independent wind-tunnel experiments with the perpendicular approaching flow, and the results showed that all the $k-\varepsilon$ models (the standard $k-\varepsilon$ model, RNG $k-\varepsilon$ model, and realizable $k-\varepsilon$ model) seemed to overrate the concentration on the leeward side of street canyon (Figures 2 and 4). Despite this discrepancy, the concentration distributions within the street canyon were reasonably simulated by the OpenFOAM in comparison with the measurements, and the standard $k-\varepsilon$ model was most accurate.

And, then, the patterns of flow and dispersion within three different kinds of street canyon configuration under the perpendicular approaching flow were studied to find out the urban-planning strategies to ease the air pollution inside the street canyon.

It is found that the width and height of building can dramatically affect the pollution level inside the street canyon. As the width or height of building increases, the pollution level increases.

Since the lower parts of the asymmetric configuration (step-up or step-down street canyon) are occupied by divergent flow fields, the nonuniform street canyon could provide better ventilation.

Finally, it should be pointed out that it is not always a perpendicular flow which dominates the actual built-up area. Therefore, the work reported here is not suitable for a particular area. More specific studies are needed to find out the urban-planning strategies to ease the air pollution for a particular area. However, even at this stage of the research, it is recommended to design a street canyon with nonuniform configurations. And for numerical study of flow and dispersion within built-up areas, the OpenFOAM can be used as a reliable tool.

\section{Conflict of Interests}

The authors declare that there is no conflict of interests regarding the publication of this paper.

\section{Acknowledgment}

This work is supported by Public Welfare Special Fund Program (Meteorology) of the Chinese Ministry of Finance under Grant no. GYHY201106033.

\section{References}

[1] T. R. Oke, "Street design and urban canopy layer climate," Energy and Buildings, vol. 11, no. 1-3, pp. 103-113, 1988.

[2] P. G. Mestayer, P. Durand, P. Augustin et al., "The urban boundary-layer field campaign in Marseille (UBL/CLUESCOMPTE): set-up and first results," Boundary Layer Meteorology, vol. 114, no. 2, pp. 315-365, 2005.

[3] S. Xie, Y. Zhang, L. Qi, and X. Tang, "Spatial distribution of traffic-related pollutant concentrations in street canyons," Atmospheric Environment, vol. 37, no. 23, pp. 3213-3224, 2003.

[4] K. J. Allwine, J. H. Shinn, G. E. Streit, K. L. Clawson, and M. Brown, "Overview of Urban 2000: a multiscale field study of dispersion through an urban environment," Bulletin of the American Meteorological Society, vol. 83, no. 4, pp. 521-536, 2002.

[5] X. X. Li, C. H. Liu, D. Y. C. Leung, and K. M. Lam, "Recent progress in CFD modelling of wind field and pollutant transport in street canyons," Atmospheric Environment, vol. 40, no. 29, pp. 5640-5658, 2006.

[6] J. J. Baik, R. S. Park, H. Y. Chun, and J. J. Kim, "A laboratory model of urban street-canyon flows," Journal of Applied Meteorology, vol. 39, no. 9, pp. 1592-1600, 2000.

[7] X. X. Li, D. Y. C. Leung, C. H. Liu, and K. M. Lam, "Physical modeling of flow field inside urban street canyons," Journal of Applied Meteorology and Climatology, vol. 47, no. 7, pp. 20582067, 2008.

[8] Y. A. Gayev and E. Savory, "Influence of street obstructions on flow processes within urban canyons," Journal of Wind Engineering and Industrial Aerodynamics, vol. 82, no. 1-3, pp. 89-103, 1999.

[9] R. N. Meroney, M. Pavageau, S. Rafailidis, and M. Schatzmann, "Study of line source characteristics for 2-D physical modelling of pollutant dispersion in street canyons," Journal of Wind Engineering and Industrial Aerodynamics, vol. 62, no. 1, pp. 3756, 1996.

[10] P. Kastner-Klein, E. Fedorovich, and M. W. Rotach, "A wind tunnel study of organised and turbulent air motions in urban street canyons," Journal of Wind Engineering and Industrial Aerodynamics, vol. 89, no. 9, pp. 849-861, 2001.

[11] H. Liu, B. Liang, F. Zhu, B. Zhang, and J. Sang, "A laboratory model for the flow in urban street canyons induced by bottom heating," Advances in Atmospheric Sciences, vol. 20, no. 4, pp. 554-564, 2003.

[12] J. J. Baik and J. J. Kim, "A numerical study of flow and pollutant dispersion characteristics in urban street canyons," Journal of Applied Meteorology, vol. 38, no. 11, pp. 1576-1589, 1999.

[13] V. D. Assimakopoulos, H. M. ApSimon, and N. Moussiopoulos, "A numerical study of atmospheric pollutant dispersion in different two-dimensional street canyon configurations," Atmospheric Environment, vol. 37, no. 29, pp. 4037-4049, 2003.

[14] N. Koutsourakis, J. G. Bartzis, and N. C. Markatos, "Evaluation of Reynolds stress, $\mathrm{k}-\varepsilon$ and RNG $\mathrm{k}-\varepsilon$ turbulence models in street canyon flows using various experimental datasets," Environmental Fluid Mechanics, vol. 12, no. 4, pp. 379-403, 2012.

[15] E. Solazzo, X. Cai, and S. Vardoulakis, "Improved parameterisation for the numerical modelling of air pollution within an urban street canyon," Environmental Modelling and Software, vol. 24, no. 3, pp. 381-388, 2009.

[16] Y. O. Nelso and D. Y. C. Leung, "Effects of building aspect ratio, diurnal heating scenario, and wind speed on reactive pollutant 
dispersion in urban street canyons," Journal of Environmental Sciences, vol. 24, no. 12, pp. 2091-2103, 2012.

[17] Y. W. Zhang, Z. L. Gu, S. C. Lee, T. M. Fu, and K. F. Ho, "Numerical simulation and in Situ investigation of fine particle dispersion in an actual deep street canyon in Hong Kong," Indoor and Built Environment, vol. 20, no. 2, pp. 206-216, 2011.

[18] J. J. Kim and J. J. Baik, "Effects of inflow turbulence intensity on flow and pollutant dispersion in an urban street canyon," Journal of Wind Engineering and Industrial Aerodynamics, vol. 91, no. 3, pp. 309-329, 2003.

[19] A. T. Chan, E. S. P. So, and S. C. Samad, "Strategic guidelines for street canyon geometry to achieve sustainable street air quality," Atmospheric Environment, vol. 35, no. 24, pp. 5681-5691, 2001.

[20] S. D. Sabatino, R. Buccolieri, B. Pulvirenti, and R. E. Britter, "Flow and pollutant dispersion in street canyons using FLUENT and ADMS-Urban," Environmental Modeling and Assessment, vol. 13, no. 3, pp. 369-381, 2008.

[21] X. X. Li, C. H. Liu, and D. Y. C. Leung, "Large-eddy simulation of flow and pollutant dispersion in high-aspect-ratio urban street canyons with wall model," Boundary Layer Meteorology, vol. 129, no. 2, pp. 249-268, 2008.

[22] Y. C. Miao, S. H. Liu, B. C. Chen et al., "Simulating urban flow and dispersion in Beijing by coupling CFD model with WRF model," Advance in Atmospheric Sciences, vol. 30, no. 6, pp. 1663-1678, 2013.

[23] B. C. Chen, S. H. Liu, Y. C. Miao et al., "Construction and validation of an urban area flow and dispersion model on building scales," Acta Meteorologica Sinica, vol. 27, no. 6, pp. 923-941, 2013.

[24] J. H. Ferziger and M. Peric, Computational Methods for Fluid Dynamics, Springer, Berlin, Germany, 3rd edition, 2001.

[25] M. Jicha, J. Pospisil, and J. Katolicky, "Dispersion of pollutants in street canyon under traffic induced flow and turbulence," Environmental Monitoring and Assessment, vol. 65, no. 1-2, pp. 343-351, 2000.

[26] S. B. Pope, Turbulent Flows, Cambridge University Press, Cambridge, UK, 2001.

[27] S. Rafailidis, "Influence of building areal density and roof shape on the wind characteristics above a town," Boundary Layer Meteorology, vol. 85, no. 2, pp. 255-271, 1997.

[28] C. Gromke, R. Buccolieri, S. Di Sabatino, and B. Ruck, "Dispersion study in a street canyon with tree planting by means of wind tunnel and numerical investigations-evaluation of CFD data with experimental data," Atmospheric Environment, vol. 42, no. 37, pp. 8640-8650, 2008.

[29] P. Moonen, C. Gromke, and V. Dorer, "Performance assessment of Large Eddy Simulation (LES) for modeling dispersion in an urban street canyon with tree planting," Atmospheric Environment, vol. 75, pp. 66-76, 2013.

[30] J. Franke, A. Hellsten, H. Schlünzen et al., "Best practice guideline for the CFD simulation of flows in the urban environment," COST 732: Quality Assurance and Improvement of Microscale Meteorological Models, 2007. 

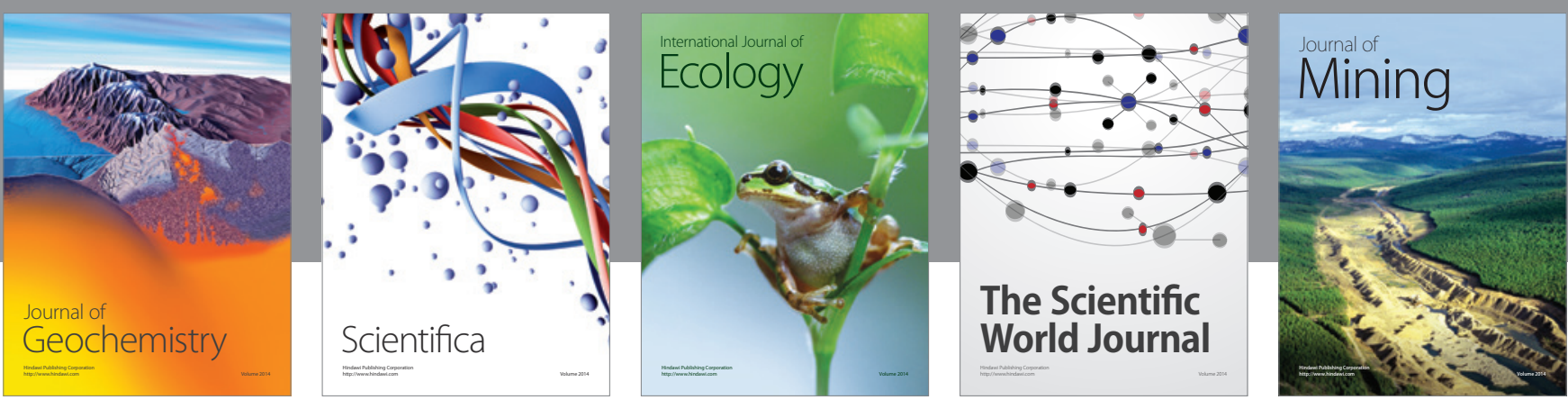

The Scientific World Journal
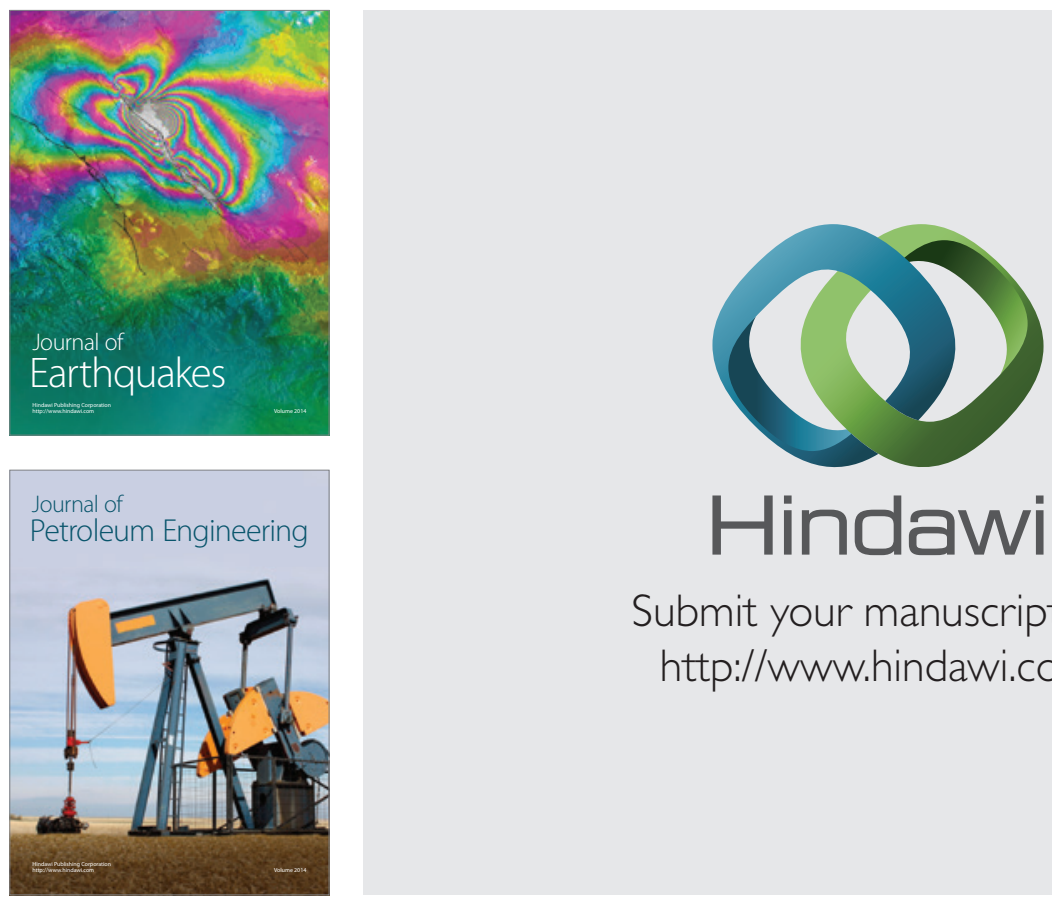

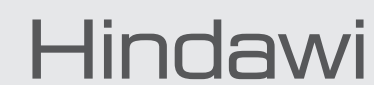

Submit your manuscripts at

http://www.hindawi.com
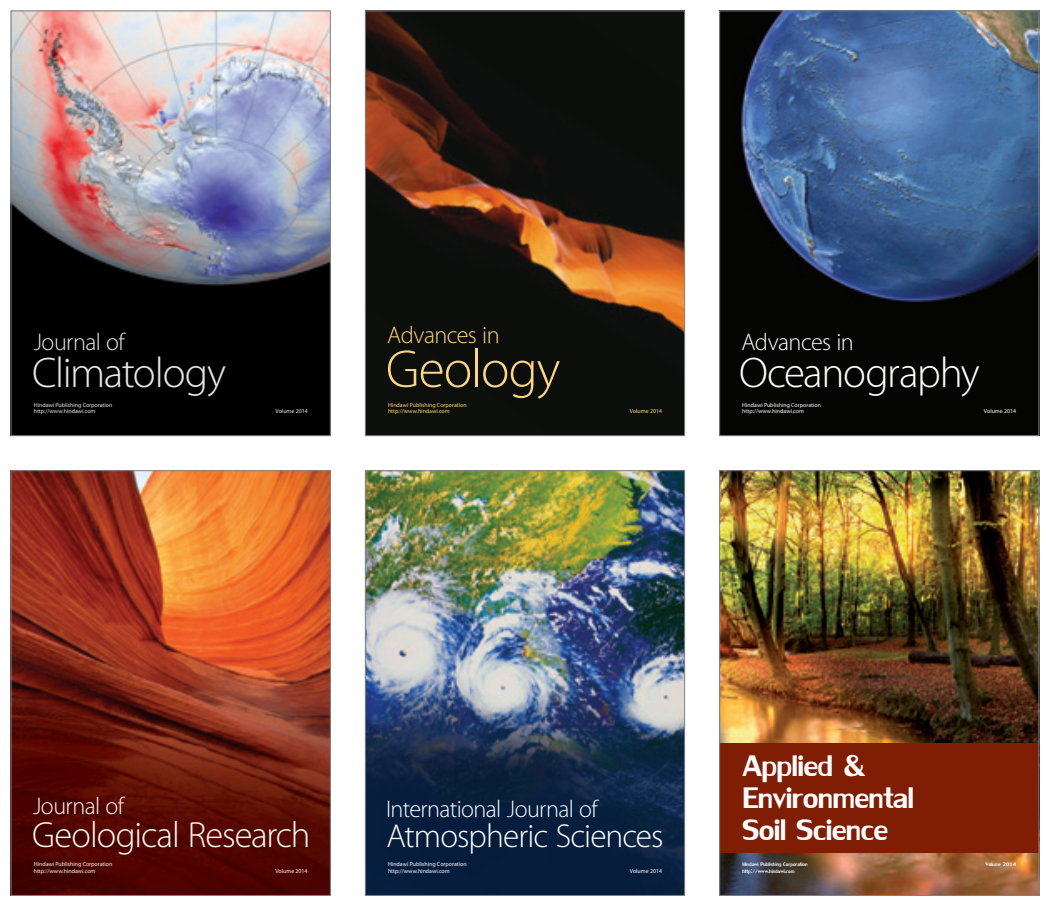
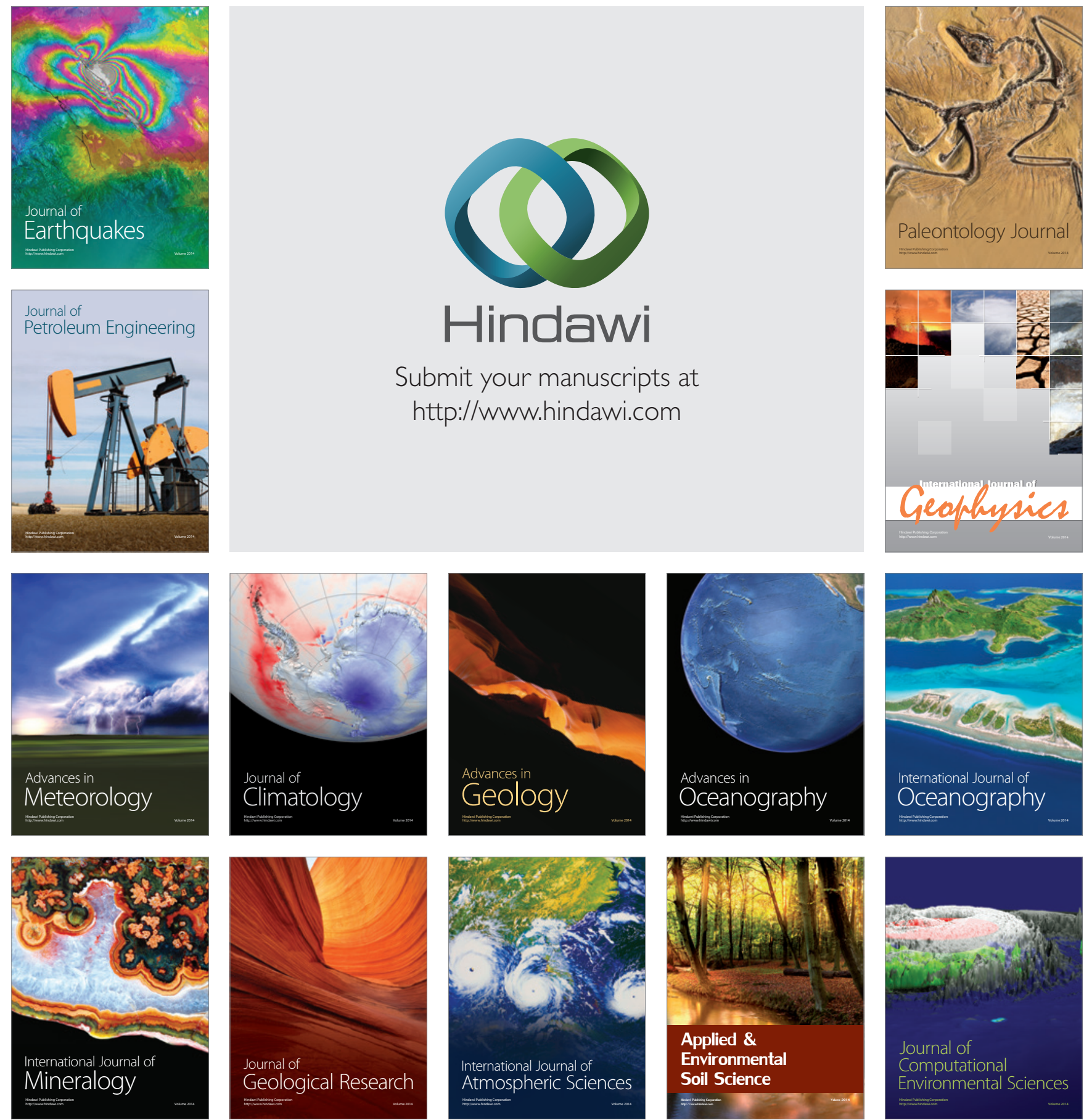